\title{
An in vivo, neuron-specific approach for pairing translational and epigenetic signatures of early-life exercise.
}

\author{
Raus AM*1, Fuller TD*2, Nelson NE*1, Bayat $A^{2}$, Valientes SDA², Ivy $\mathrm{AS}^{1,2,3,4}$ \\ *These authors contributed equally to the work. \\ ${ }^{1}$ Department of Physiology and Biophysics, \\ 2Department of Pediatrics University of California- Irvine School of Medicine, \\ Irvine, CA, \\ ${ }^{3}$ Department of Anatomy and Neurobiology University of California- Irvine School \\ of Medicine, Irvine, CA, \\ University of California- Irvine School of Medicine, Irvine, CA \\ ${ }^{4}$ Division of Neurology, Children's Hospital Orange County, Orange, CA \\ Correspondence to aivy@hs.uci.edu
}




\begin{abstract}
Aerobic exercise promotes physiological and molecular adaptations in neurons to influence brain function and behavior. The most well studied neurobiological consequences of exercise are those which underlie exercise-induced improvements in hippocampal memory, including the expression and regulation of the neurotrophic factor $B d n f$. Whether aerobic exercise taking place during early-life periods of postnatal brain maturation has similar impacts on gene expression and its regulation remains to be investigated. Using unbiased next-generation sequencing we characterize gene expression programs and their regulation by specific, memory-associated histone modifications during juvenile-adolescent voluntary exercise (ELE). Traditional transcriptomic and epigenomic sequencing approaches have either used heterogeneous cell populations from whole tissue homogenates or flow cytometry for single cell isolation to distinguish cell types / subtypes. These methods fall short in providing cell-type specificity without compromising sequencing depth or procedure-induced changes to cellular phenotype. In this study, we use simultaneous isolation of translating mRNA and nuclear chromatin from a neuron-enriched cell population to more accurately pair ELE-induced changes in gene expression with epigenetic modifications. We employ a line of transgenic mice expressing the NuTRAP (Nuclear Tagging and Translating Ribosome Affinity Purification) cassette under the $E m x 1$ promoter allowing for brain cell-type specificity. We then developed a technique that combines nuclear isolation using Isolation of Nuclei TAgged in Specific Cell Types (INTACT) with Translating Ribosomal Affinity Purification (TRAP) methods to determine cell type-specific epigenetic modifications influencing gene expression programs from a population of $E m x 1$ expressing hippocampal neurons. Data from RNA-seq and CUT\&RUN-seq were coupled to evaluate histone modifications influencing the expression of translating mRNA in neurons after early-life exercise (ELE). We also performed separate INTACT and TRAP isolations for validation of our protocol and demonstrate similar molecular functions and biological processes implicated by gene ontology (GO) analysis. Finally, as prior studies use tissue from opposite brain hemispheres to pair transcriptomic and epigenomic data from the same rodent, we
\end{abstract}


take a bioinformatics approach to compare hemispheric differences in gene expression programs and histone modifications altered by by ELE. Our data reveal transcriptional and epigenetic signatures of ELE exposure and identify novel candidate gene-histone modification interactions for further investigation. Importantly, our novel approach of combined INTACT/TRAP methods from the same cell suspension allows for simultaneous transcriptomic and epigenomic sequencing in a cell-type specific manner. 


\section{Introduction}

Environmental experiences engage epigenetic mechanisms to modulate gene expression and cell function in post-mitotic neurons [1,2]. Histone modifications and DNA methylation are particularly important for neuronal adaptation to environmental signals by altering gene transcription and synaptic function $[3,4]$. Behavioral outputs such as stress susceptibility, reward seeking, and long-term memory have been shown to result from changes to chromatin accessibility and gene expression in neurons [5-9]. In addition to this, the neuronal chromatin landscape undergoes waves of epigenetic modifications as a function of brain maturation itself [10-12]. Postnatal periods of heightened sensitivity to environmental stimuli can lead to lasting changes to cellular function, and may result from temporally specific epigenetic mechanisms in the developing brain [13, 14]. How epigenetic mechanisms in postmitotic neurons are influenced by early-life experiences to inform long-term function is beginning to be explored. Identifying gene regulatory mechanisms involved in modulating cell function during sensitive windows of brain development can aid in understanding how early-life experiences inform long-term behavioral outcomes.

Aerobic exercise has generally been found to enhance performance on cognitive tasks involving the hippocampus in both adult humans and animal models $[15,16]$. The type, timing, and duration of exercise exposure matters with regard to whether it has persistent impact on hippocampal function [17-19]. Findings in rodents demonstrate that voluntary exercise exposure or treatment with HDAC3 inhibition improves hippocampal memory function [20-22]. Although the majority of these studies have been performed in adults, more recent work suggests that the effects of exercise on hippocampal memory and the associated alterations in neurotrophic factor expression, synaptic plasticity, and neurogenesis are similar in juvenile and adolescent periods [17, 23-26]. Previous work in our lab showed that voluntary wheel running for either one week (postnatal days (P) 21-27) or three weeks (P21-41) led to enabled hippocampal long-term memory (LTM) in response to a subthreshold learning experience when tested on P42. This was associated with increased CA1 LTP in the three-week exercise group, as well as modulations to CA1 
synaptic physiology in both the one-week and three-week exercisers [17]. Notably, the effects of exercise during P21-27 lasted at least two weeks after exercise cessation, which may be longer than the influence of exercise on adult hippocampal function [27]. This finding suggests that exercise exposure during a sensitive period of postnatal hippocampal development has the potential to persistently impact hippocampal function.

The goal of this study is to identify novel programs of neuronal gene expression and their epigenetic regulation resulting from exercise during early life. In order to do this, we developed a transgenic mouse line and experimental protocol to simultaneously obtain epigenetic and transcriptional information from Emx1expressing neurons of the hippocampus. By crossing the Emx1-Cre transgenic line with mice expressing the NuTRAP (Nuclear Tagging and Translating Ribosome Affinity Purification [28]) cassette (termed Emx1-NuTRAP mice) we are able to simultaneously isolate whole nuclei and translating mRNA from a neuron-enriched population of cells. Using the same tissue sample, we combine the first steps of the INTACT (Isolation of Nuclei TAgged in specific Cell Types[29]) and TRAP (Translating Ribosomal $\underline{\text { Affinity }}$ Purification [30]) protocols and obtain high-quality DNA and RNA yields for downstream sequencing procedures. Juvenile Emx1NuTRAP mice underwent three weeks of early-life exercise during P21-41 (hereafter referred to as "ELE") in order to determine the epigenetic regulation of exercise-induced changes to gene expression and cell function. Using novel technical approaches, this study uncovers hippocampal gene expression programs and associated histone modifications that are driven by voluntary wheel running during the juvenile and adolescent developmental periods.

\section{Results}

\section{“Emx1-NuTRAP" mouse allows for simultaneous isolation of nuclear chromatin and translating mRNA from a single population of hippocampal neurons.}

Our goal was to obtain translating mRNA and nuclear DNA from a single population of neurons in order to couple transcriptional network changes with 
alterations in histone modifications resulting from ELE. We therefore crossed NuTRAP reporter [28] with Emx1- Cre [31,32] mouse lines to generate Emx1- Cre; NuTRAP transgenic progeny for this study (Figure 1A). In the presence of a loxP siteflanked sequence, Emx1-Cre facilitates recombination in approximately $88 \%$ of neurons in the neocortex and hippocampus, and in less than $2 \%$ of GABAergic inhibitory interneurons in these regions [31]. Emx1- Cre also targets neural progenitor cells and can be expressed in mature astrocytes in striatum [33]. Of note, we exclusively used Emx1-Cre female mice in our breeding schemes given that the Cre -recombinase has been reported to be expressed in a subset of male germline cells [34].

We sought to validate neuron-specific expression of the NuTRAP cassette. First, immunohistochemistry of Emx1-NuTRAP hippocampal slices show distinct GFP and mCherry expression in CA1 and CA3 pyramidal neurons (Figure 1B-C) as well as the granule cell layer of the dentate gyrus (DG) (Figure 1D). Furthermore, mCherry positive nuclei remained intact and bound to the auto-fluorescent magnetic beads after our modified nuclear isolation procedure (Supplemental Figure 1A). As Emx1-expressing neural stem cells can become astrocytes [31], we sought to determine whether a significant number of mature astrocytes were obtained in the population of isolated cells. Flow cytometry experiments using THY1 cell surface marker to label neurons and $S 100 \beta$ as an astrocytic marker revealed a distinct population of cells double positive for GFP and THY-1, whereas a S100 $\beta$ and GFP double positive cell population was absent (Figure 1E).

To perform simultaneous isolation of labeled ribosomes and nuclei from the same homogenate, hippocampal tissue was dissected from both brain hemispheres, combined, and homogenized in one sample tube (Figure 1A). We then performed the INTACT and TRAP methods on isolated samples by starting the protocol with the beginning steps from the INTACT procedure, modified to include cycloheximide. Cycloheximide works rapidly to inhibit protein synthesis and is used for maintaining crosslinks between translating mRNA and ribosomal subunits during purification in the traditional TRAP method. Despite the presence of cycloheximide, nuclear morphology from Emx1-expressing neurons is overall unchanged 
(Supplemental Figure 1A). Additionally, a Bioanalyzer was used to determine if there were cycloheximide-induced double-stranded DNA breaks in our nuclear preparation that could interfere with downstream DNA sequencing applications (such as ATAC, CUT\&RUN, or CUT\&Tag). We found no evidence of DNA double strand breaks generating fragments of less than $1 \mathrm{~kb}$ (Supplemental Figure 2B). Following magnetic purification of biotin-labeled nuclei, the supernatant fraction was removed and taken through TRAP, while the pelleted nuclei were processed through the remaining steps of INTACT (see Methods). Combining bilateral hippocampi from a single mouse yielded TRAP-isolated RNA of high quality and sufficient concentration for sequencing (RIN > 8 for all samples, average yield RNA = 14.36688 ng/ul; Supplemental Table 1). INTACT-isolated nuclei were further processed using the CUT\&RUN (leavage $\underline{\text { Under }}$ Targets and $\underline{\text { Release }}$ Using Nuclease[35]) method to isolate antibody-specific protein-DNA interactions for downstream DNA sequencing. The resulting DNA libraries were of high quality and concentration when using specific antibodies (H4K8ac: average size = $1197 \mathrm{bp}$, average concentration $=127.8 \mathrm{nM}$; H3K27me3: average size $=1056 \mathrm{bp}$, average concentration $=139.0 \mathrm{nM}$; Supplemental Table 1). In contrast, the resulting DNA libraries using the non-specific IgG control were of substantially lower concentrations (IgG: average size $=1075 \mathrm{bp}$, average concentration $=16.3 \mathrm{nM}$; Supplemental Table 1) further indicating that nuclear DNA from both isolations was of high starting quality.

In order to confirm our TRAP-isolated hippocampal mRNA came primarily from excitatory neurons, we took a bioinformatics approach to assess for neuronal gene enrichment. Specifically, we compared RNA-seq data from whole dorsal hippocampal tissue of wild-type mice to our TRAP-isolated RNA-seq data. We found that TRAP-isolated mRNA had significant enrichment of several neuronal genes including Dlg4, Thy1, Eno2, and Syt4, as well as a significant reduction in astrocytic, microglial, oligodendrocyte, and inhibitory neuron genes (Figure 1F). There was a relative expression increase of the glial fibrillary astrocytic protein (GFAP) in our TRAP samples. This gene can also be expressed in neural stem populations that were present in our whole hippocampus samples, so this finding may be a reflection 
of the neural stem cell population of the dentate gyrus captured as part of our TRAP isolation [36, 37]. Overall, these findings suggest that the Emx1-NuTRAP mouse model is a valid tool for neuron-enriched isolation of sequencing-grade, translating mRNA and nuclear chromatin from the same cell homogenate. 
bjoRxiv preprint doi: https://doi.org/10.1101/2021.12.23.473936; this version posted December 23, 2021. The copyright holder for this preprint Figure 1 (which was not certified by peer review) is the author/funder. All rights reserved. No reuse allowed without permission.

A

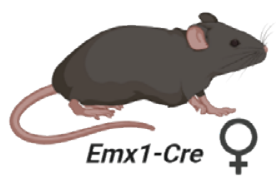

Emx1-expressing cells
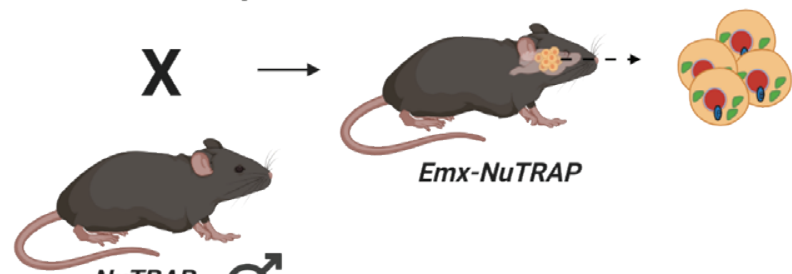

Emx-NuTRAP

NUTRAP $\Upsilon^{T}$
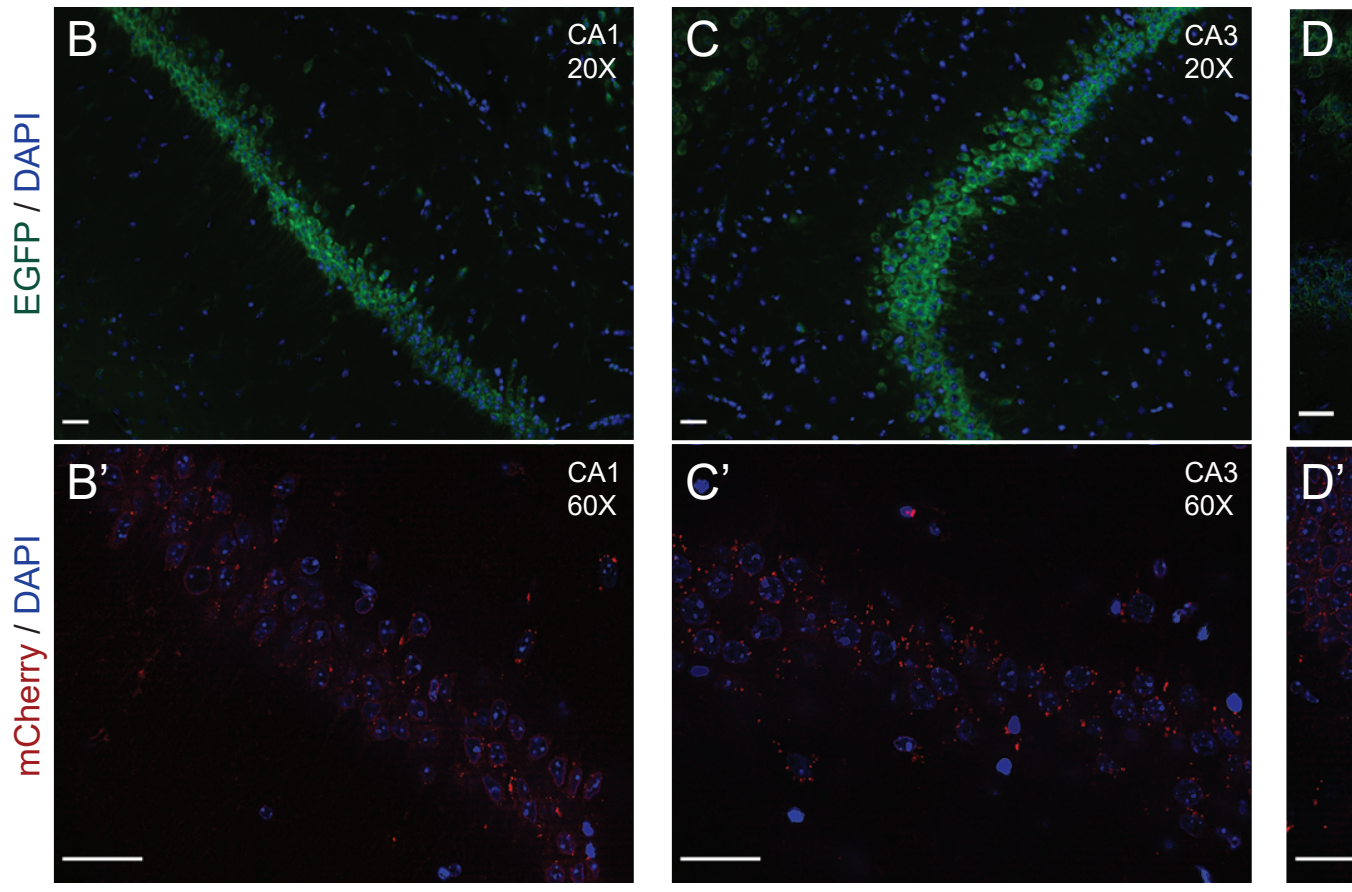

RNA-Seq

INTACT isolation

CUT\&RUN-Seq
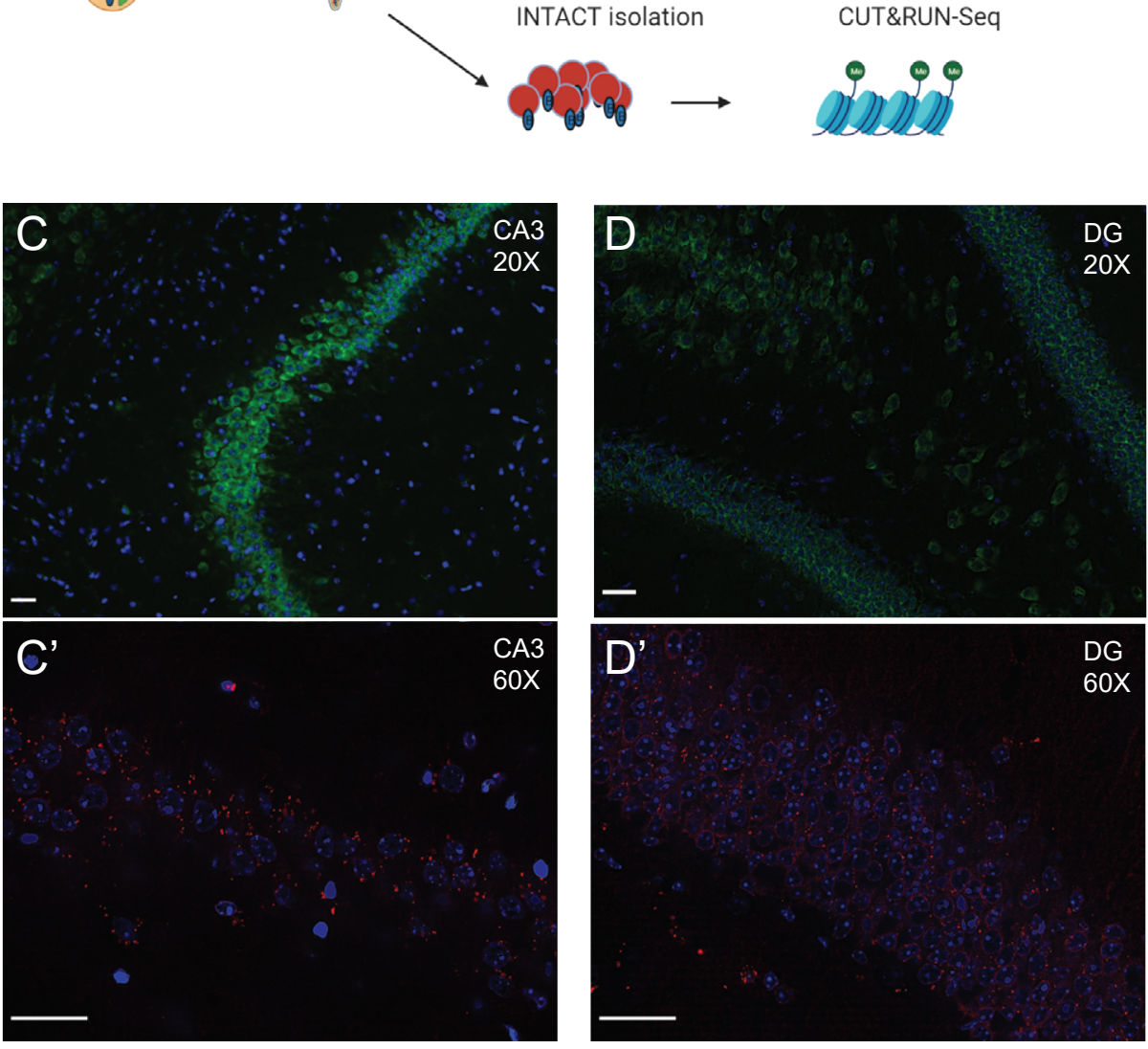

E
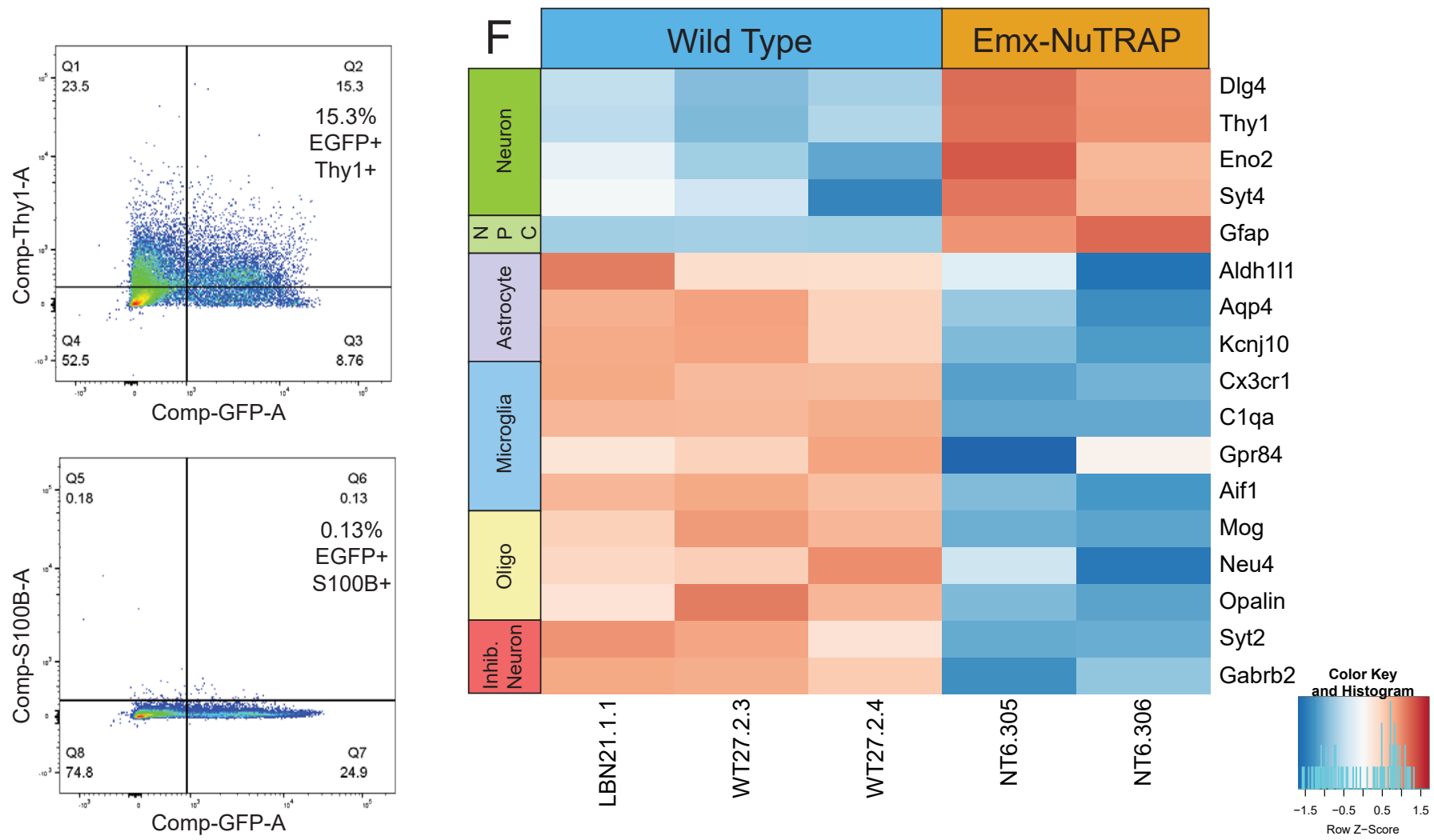
Figure 1. Generation and validation of the Emx1-NuTRAP mouse (A) Schematic representation of the Emx1-NuTRAP mouse generation and workflow of the simultaneous isolation protocol. (B) Immunofluorescence imaging of the CA1 region of the hippocampus at 20x objective (B) and 60x objective (B') after incubation with an mCherry antibody overnight. (C) Immunofluorescence imaging of the CA3 and CA2 region of the hippocampus at 20x objective (C) and 60x objective (C') after incubation with an mCherry antibody overnight. (D) Immunofluorescence imaging of the DG region of the hippocampus at 20x objective (D) and 60x objective (D') after incubation with an mCherry antibody overnight. Scale bars are set to $50 \mu \mathrm{m}$ for all images (B-D). (E) Flow cytometry for EGFP, THY-1, and S100 3 (15.3\% of cells THY-1+/EGFP+ and $0.13 \%$ of cells S100ß+/EGFP+). (F) Heatmap of differentially expressed neuronal and other cell type markers from RNA-seq data comparing TRAP isolated RNA from our Emx1-NuTRAP mice and previously generated dorsal hippocampal tissue RNA from wild type. 


\section{Performing INTACT/TRAP on the same or separate cell suspensions yields highly comparable RNA-seq and CUT\&RUN-seq results.}

Previous methods for performing INTACT and TRAP using NuTRAP mice have taken separate tissue homogenates for each procedure $[28,38]$. In this study, we performed simultaneous isolation of nuclei and RNA from a single tissue homogenate containing bilateral hippocampi (referred to as "simultaneous isolations"). To determine if our approach of simultaneous INTACT and TRAP isolations does not interfere with data interpretation, we also performed separate INTACT and TRAP from hippocampal tissue obtained from one brain hemisphere for each method, counterbalancing for left versus right (we refer to this protocol as "separate isolations"). A Bioanalyzer was used to determine the amount and quality of the RNA obtained from each type of isolation (Separate isolations: average RNA concentration $=7.805 \mathrm{ng} / \mathrm{ul}$, average $\mathrm{RIN}=9.5$; Simultaneous isolations: average RNA concentration = $14.367 \mathrm{ng} / \mathrm{ul}$, average RIN = 9.3; Supplemental Table 1). The average RNA yield from the separate isolations (using a unilateral hippocampus) was approximately equal to half of the average yield of the simultaneous isolations (which combined bilateral hippocampi; Supplemental Table 1). Similarly, the final library concentrations for the separately isolated IgG CUT\&RUN libraries were also approximately half the concentration of the simultaneous (Average simultaneous: 24.2nM, Average separate: $11 \mathrm{nM}$; Supplemental Table 1). We interpret this to mean that nuclear DNA was fully intact in the simultaneous isolation because we did not obtain substantially more than double the concentration in the simultaneous vs separate isolations. Unilateral hippocampal homogenates yielded sufficient sequencing concentrations and quality to allow for library preparations from individual mice (Supplemental Table 1).

To determine if normalized RNA-seq counts were similar between the two isolation methods, we performed a Spearman's Rank-Order Correlation between the datasets from sedentary mice. The two RNA-seq datasets were found to be highly correlated, with $R$ values greater than 0.5 and $p$ value less than $2.2 \times 10^{-16}(R=1$, $\mathrm{p}<2.2 \times 10^{-16}$; Figure 2A). CUT\&RUN sequencing was used to identify genomic regions interacting with either $\mathrm{H} 4 \mathrm{~K} 8 \mathrm{ac}$, an activating histone post-translational modification 
(PTM), or H3K27me3, a generally repressive histone PTM. We again applied Spearman's Rank-Order Correlation to understand whether the simultaneous vs separate INTACT isolation methods could influence CUT\&RUN peak distribution. We compared normalized count data for CUT\&RUN peaks across a representative chromosome (chromosome 2). We binned 100bp increments along the entire chromosome from the simultaneous and separate isolations using datasets generated from sedentary mice. Normalized sequencing counts, which reflected reads assigned to binned genomic regions along chromosome 2 , were highly similar between conditions (H4K8ac: $\mathrm{R}=0.65, \mathrm{p}<2.2 \times 10^{-16}$, H3K27me3: $\mathrm{R}=0.81, \mathrm{p}<2.2 \times 10^{-16}$; Figure $2 \mathrm{~B}-\mathrm{C}$ ). Taken together, these experiments suggest that translating mRNA and nuclear DNA isolated from hippocampal homogenates using either simultaneous or separate isolation procedures are comparable in terms of quality, concentration, functional characterization, and normalized sequencing reads.

We next wanted to determine if the different isolation methods could bias resulting gene expression on the basis of gene length. When plotting gene length against log fold change of gene expression, we see a similar distribution pattern of ELE-induced differentially expressed hippocampal genes (DEGs) between the simultaneous and separate methods (Figure 2D-E). We then compared the ELEinduced DEGs obtained using either the separate or the simultaneous isolation methods. Although many of the genes did not overlap (Figure 2F-G), most Gene Ontology (GO) terms did (14/20 GO terms for upregulated genes and 15/17 for downregulated genes; Figure 2H-K, Supplemental Table 2). 
bioRxiv preprint doi: https://doi.org/10.1101/2021.12.23.473936; this version posted December 23, 2021. The copyright holder for this preprint Figure 2(which was not certified by peer review) is the author/funder. All rights reserved. No reuse allowed without permission.

A spearman Correlation: RNA Expression

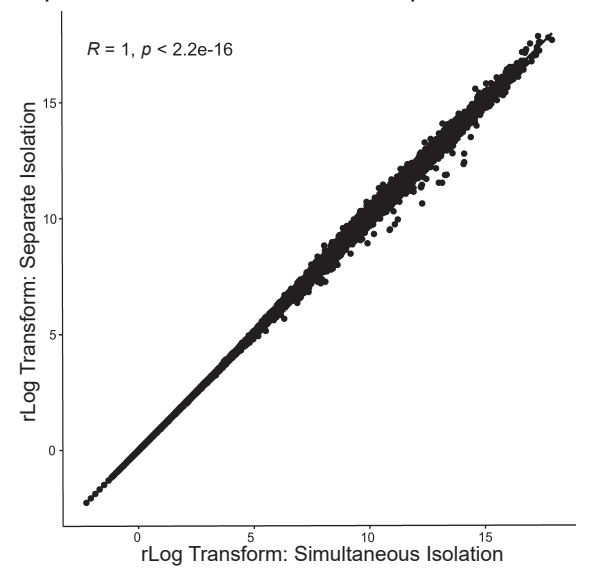

D

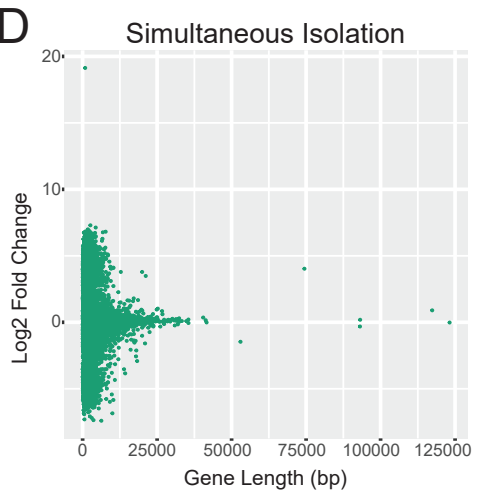

B

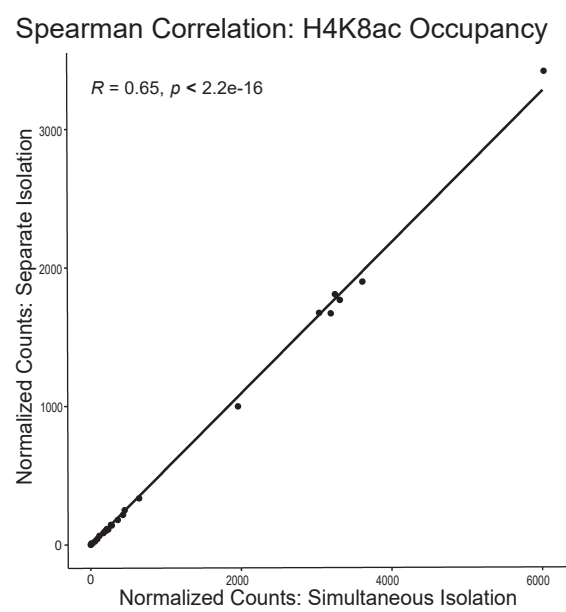

Spearman Correlation: H3K27me3 Occupancy

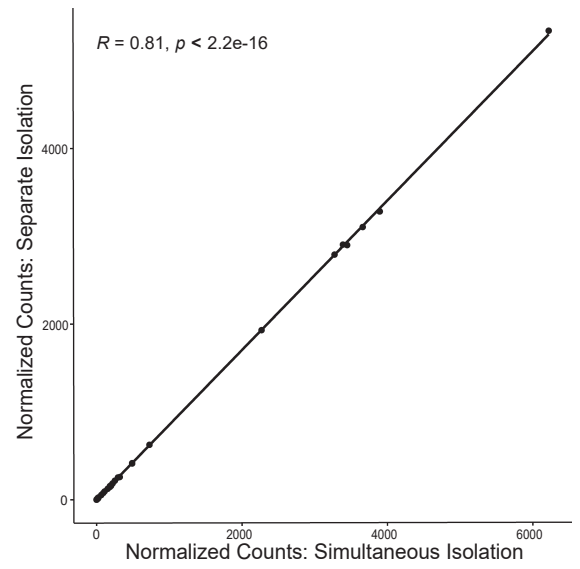

$\mathrm{E}$

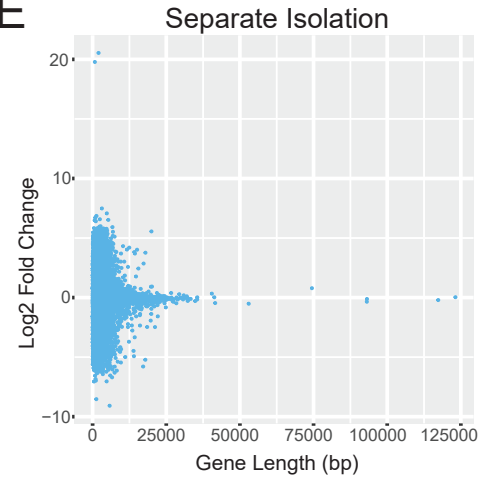

$\mathrm{F}$

Simultaneous

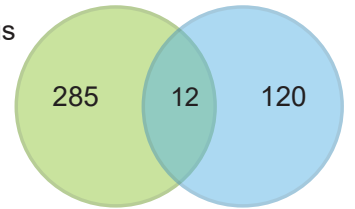

G

RNA Expression - Downregulated after ELE

Simultaneous

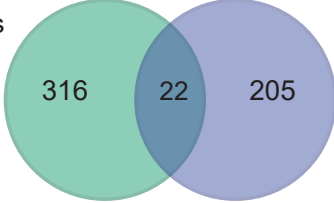

GO: Biological Processes - Downregulated after ELE

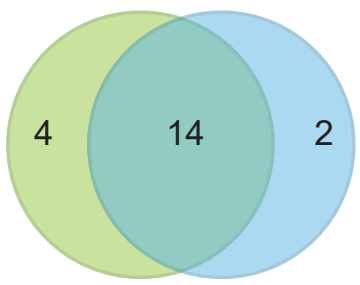

$J$

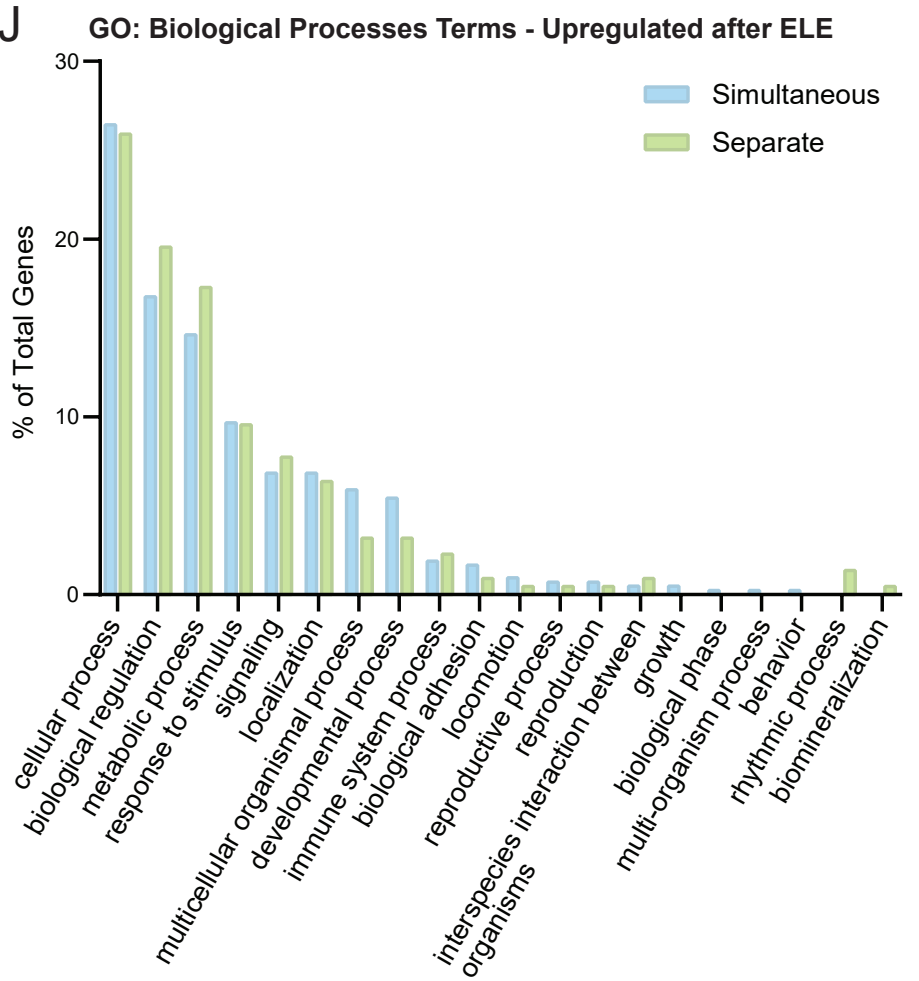

Separate
Simultaneous

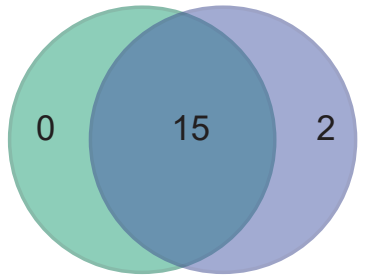

K GO: Biological Processes Terms - Downregulated after ELE

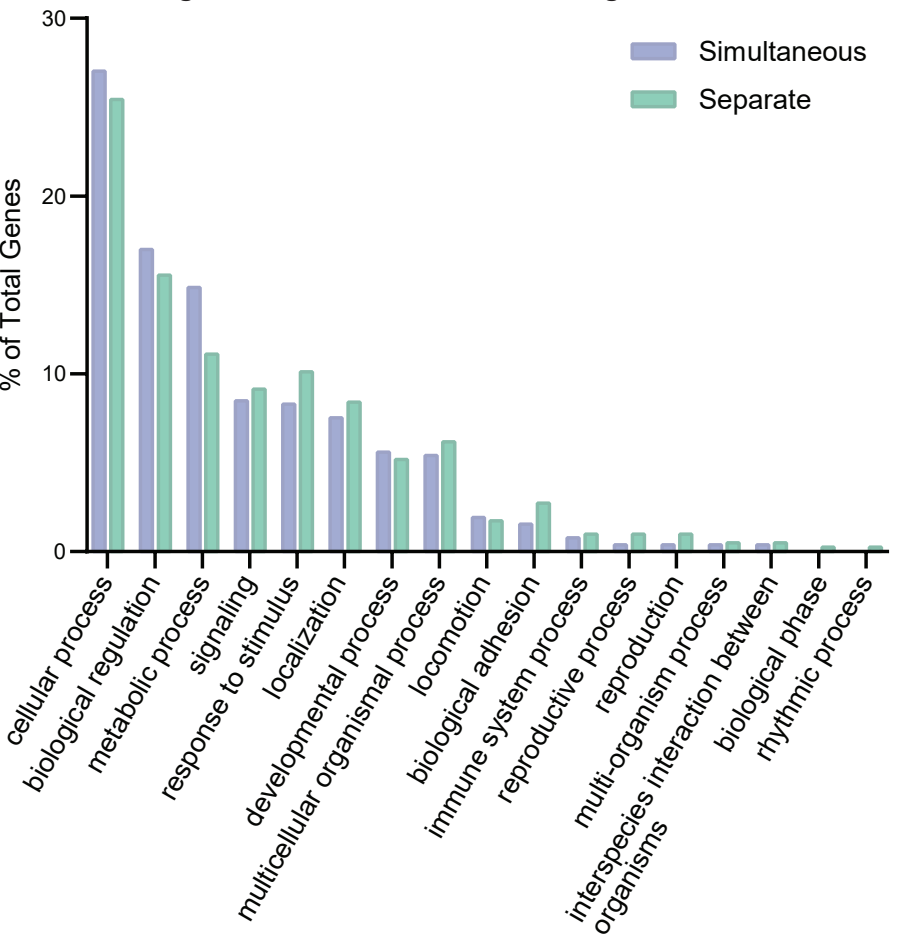




\section{Figure 2. Simultaneous and separate isolations of DNA and RNA from Emx1-}

NuTRAP mice are comparable. (A) Spearman Correlation for RNA expression between separately isolated RNA and simultaneously isolated RNA ( $\mathrm{n}=2$ mice for simultaneous isolations and 3 mice for separate isolations for Figures $2 \mathrm{~A}-\mathrm{E}, \mathrm{R}=1, \mathrm{p}<2.2 \times 10^{-16}$ ). (B) Spearman correlation between separate and simultaneously isolated DNA from CUT\&RUN-seq for H4K8ac with $100 \mathrm{bp}$ chromosome 2 position bins $\left(\mathrm{R}=0.65, \mathrm{p}<2.2 \times 10^{-}\right.$

${ }^{16}$ ). (C) Spearman correlation between separate and simultaneously isolated DNA from CUT\&RUN-seq for H3K27me3 with 100bp chromosome 2 position bins $(\mathrm{R}=0.81$, $\left.\mathrm{p}<2.2 \times 10^{-16}\right)$. (D) Gene length versus fold change distribution plot for simultaneously isolated RNA. (E) Gene length versus fold change distribution plot for separately isolated RNA. (F) Genes upregulated in Emx1-NuTRAP RNA-seq after ELE in either simultaneous ( $n=2$ sedentary mice and $n=3$ ELE mice) or separate isolations ( $n=3$ mice per group). $(G)$ Genes downregulated in Emx1-NuTRAP RNA-seq after ELE in either simultaneous or separate isolations. (H, I) Panther Gene Ontology: Biological Processes Venn diagram (H) and bar graph of gene distributions (I) for genes upregulated after ELE in either separate or simultaneous isolations. (J, K) Panther Gene Ontology: Biological Processes Venn diagram ( $\mathrm{J})$ and bar graph of gene distributions $(\mathrm{K})$ for genes downregulated after ELE in either separate or simultaneous isolations. 


\section{Early-life exercise promotes expression of plasticity-related genes and is associated with transcriptional regulatory pathways implicated in hippocampal memory.}

To identify the transcriptional effects of ELE in hippocampal neurons, we performed cell-type specific bulk sequencing on RNA extracted from Emx1-NuTRAP hippocampi using the simultaneous isolation approach. We compared DEGs from sedentary mice vs mice that underwent voluntary wheel running between postnatal days (p) 21-41. Using DESeq2 to assess for DEGs (>30\% expression increase with a $p$ value $<0.05$ ) we found that voluntary exercise during juvenile-adolescence significantly alters gene expression in Emx1 expressing neurons (297 upregulated and 338 downregulated genes; Figure 3A and Supplemental Table 2). Many of the genes upregulated after ELE are also known to be involved in exercise or hippocampal memory mechanisms, including BDNF [39] and NR4A1 [40]. To functionally categorize ELE-induced DEGs, we performed a Panther Gene Ontology (GO) analysis [41] focusing on the Molecular Function categorization and separated by upregulated and downregulated genes. The GO term categories with the most genes functionally assigned to them were binding, catalytic activity, molecular function regulator, transporter activity, molecular transducer activity, and structural molecule activity (Figure 3B). Many of the genes driving these results are known to be important in neuronal function (Kcna1, Slc24a4, Stxbp5l, Gabra2, and Camk2n2), neurodevelopment (Artn, Kdm7a, Sox21, Gap43, and Efna5), and hippocampal memory (Bdnf, Nr4a1 and Dusp5).

To evaluate possible transcription factors and upstream regulators implicated by ELE-induced DEGs, we applied Qiagen's Ingenuity Pathway Analysis (IPA) to our RNA-seq dataset [42]. The top six upstream regulators by significance included CREB1, KMT2A, LEF1, SMAD3, EGR1, and MITF. Each of the top five transcription factors have been implicated in hippocampal development or function (Figure 3C and Supplemental Table 3) [43-50]. The transcription factors KMT2A and LEF1 have not been previously associated with the effects of exercise on hippocampal function. Interestingly, both CREB1 and KMT2A have histone modifying properties; CREB1 through its associated CBP [51] and KMT2A through its methyltransferase activity 
[44]. LEF1 has been shown to regulate neural precursor proliferation in the hippocampus [45]. SMAD3 is critical for intermediate progenitor cell survival and negatively regulates serum insulin after exercise [46, 47]. EGR1 is an immediate early gene that recruits TET1 DNA demethylase during neural activation and development [48]. MITF was the sixth upstream regulator identified and is a transcription factor linked to autophagy mechanisms [49]. These specific transcription factors were not significantly differentially expressed in our RNA-seq dataset; however, their activity may not be linked to a change in their own expression after ELE but rather is modulated by exercise to influence programs of gene expression.

To further understand whether the transcriptional profiles resulting from ELE were enriched for specific, a priori assigned molecular functions, we performed Gene Set Enrichment Analysis (GSEA) [52]. We evaluated the "Reactome" category of functional gene sets followed by a leading edge analysis to further determine which genes were driving the significant categories (Figure 3D and Supplemental Table 4). Of the DEGs upregulated after ELE, several interesting categorizations and the genes driving them were revealed. The genes $H 3 c 4, H 2 b c 3, H 2 b c 5$, and $H 2 b c 9$ genes, which encode histone family member proteins, were driving the categories DNA methylation, HDACs deacetylate histones, and transcriptional regulation by smRNAs. Meiosis and meiotic recombination also emerged in these results which was unusual; however, leading edge analysis showed many of these genes (Atm, Blm, Msh4, and Mnd1) to be generally involved in cell-cycling processes. Exercise is well known to increase adult neurogenesis in hippocampal dentate gyrus and can explain cell cycle gene enrichment in our dataset [16]. Several nucleoporin complex genes (Nup35/42/43/50/58/62/93/107/153/210) were also identified for their associations with categories such as: gene silencing by RNA, nuclear envelope breakdown, transport of mature mRNAs derived from intronless transcripts. Nup genes form a variety of nuclear pore complexes that play critical roles in cellular processes including cell-cycle regulation, cellular differentiation, and epigenetic control [53]. 
Of the downregulated pathways identified, translation-associated categories (translation, eukaryotic translation initiation/elongation, and SRP-dependent cotranslational protein targeting to membrane) were driven by significant downregulation of ribosomal protein gene families ( $R p s, R p l, R p l p)$ and eukaryotic initiation factor 3 (eIF3) subunits. Over-expression of eIF3 subunits has been linked to Parkinson's Disease and altered expression of eIF3 has been associated with neurodevelopmental disorders [54]. Additionally, collagen genes (Col1a1, Col1a2, Col2a1, Col3a1, and Col5a1) were downregulated after ELE, leading to the identification of categories including: MET activates PTK2 signaling, MET promotes cell motility, and collagen degradation. Col1a1 and Col1a2 have been previously identified as putative aging genes that decrease in their expression after chronic exercise in adults [55]. By analyzing these enriched pathways and the networks of genes driving them, we were able to identify a number of transcriptional programs activated by ELE that could be candidates for further exploration. 
bioRxiv preprint doi: https://doi.org/10.1101/2021.12.23.473936; this version posted December 23, 2021. The copyright holder for this preprint

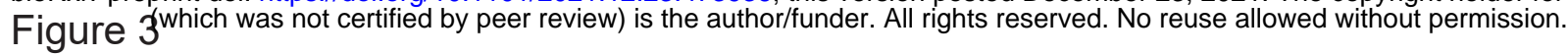

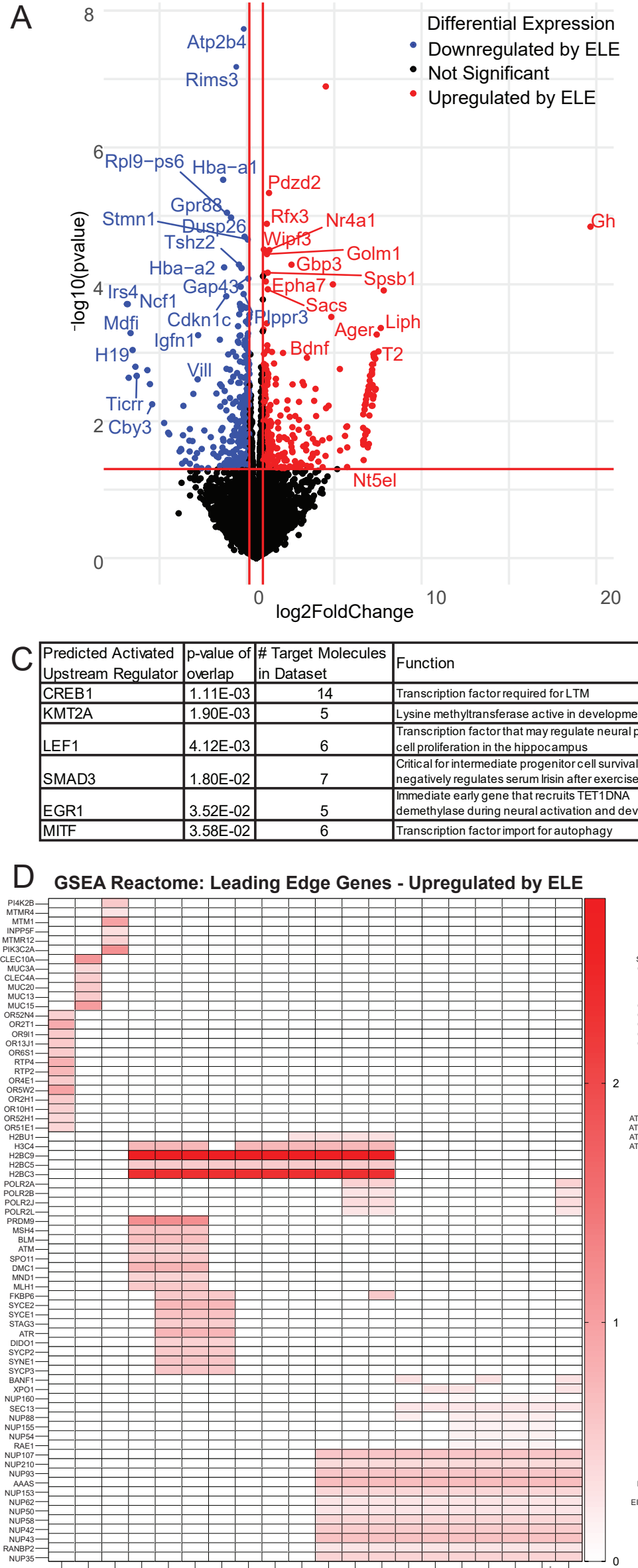

B

GO Terms: Molecular Function - Upregulated by ELE

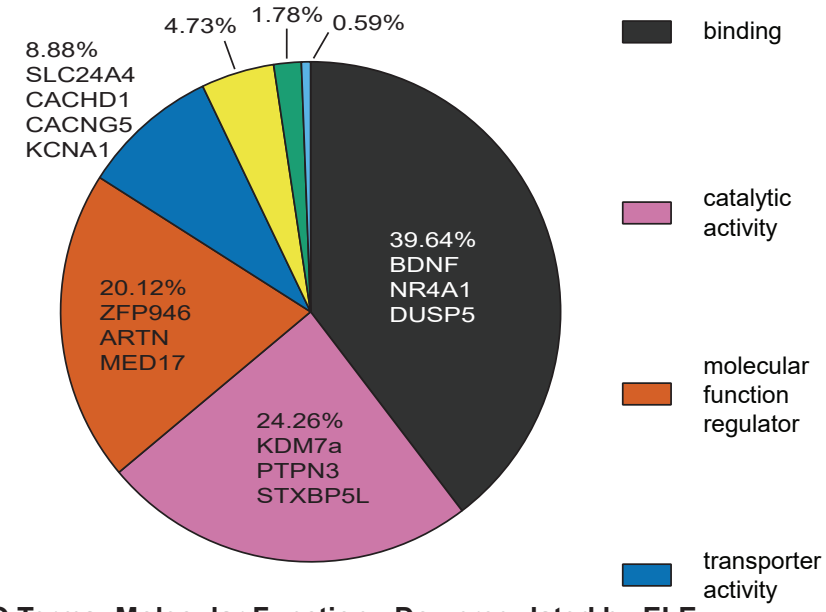

GO Terms: Molecular Function - Downregulated by ELE

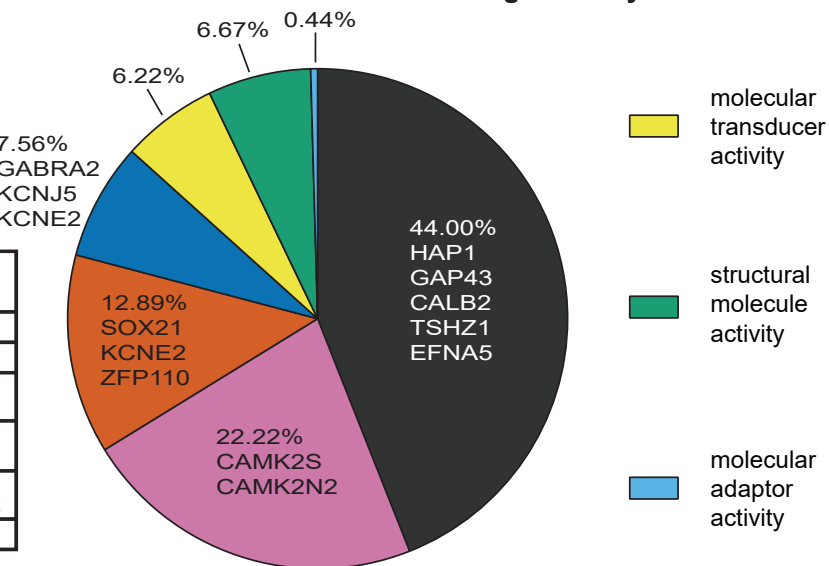

GSEA Reactome: Leading Edge Genes - Downregulated by ELE
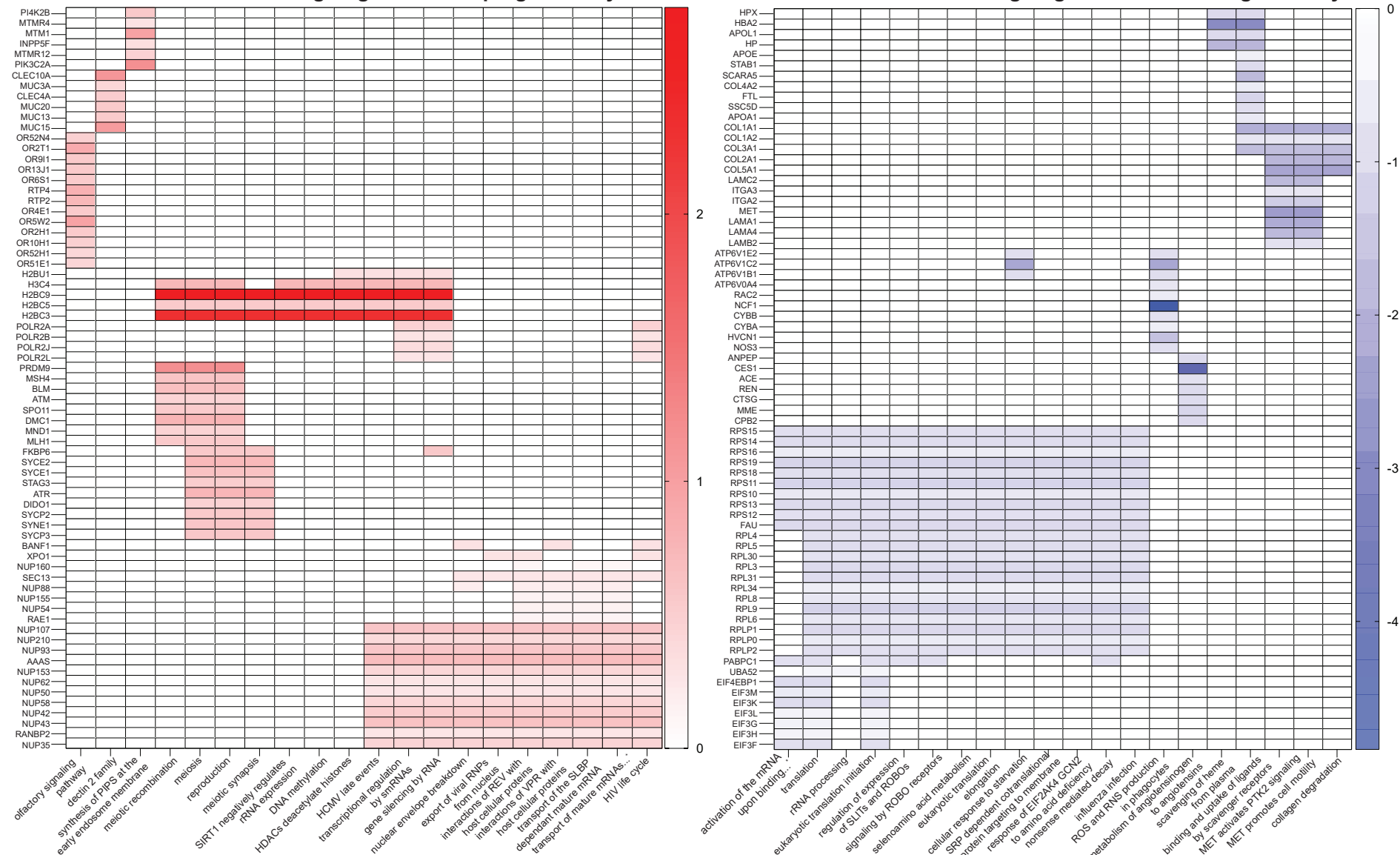


\section{Figure 3: ELE leads to transcriptomic changes in hippocampal neurons during}

adolescence. (A) Volcano plot of significantly differentially expressed genes from RNAseq on ELE versus sedentary samples ( $n=2$ sedentary mice and $n=3$ ELE mice, absolute value $\log _{2}$ fold change $>0.3785$ and p-value < 0.05). (B) Panther Gene Ontology: Molecular Function top terms by most genes assigned. (C) Top 6 upstream regulators identified by Ingenuity Pathway Analysis (IPA). (D) Representative Gene Set Enrichment Analysis: Reactome leading edge diagrams showing genes that are upregulated (D) or downregulated (D') in ELE, and their enrichment. *Abbreviated terms in (D) transport of mature mRNAs derived from intronless transcripts, (D') activation of the mRNA upon binding of the cap binding complex and EIFs and subsequent binding to 43S. 


\section{Neuronal H4K8ac is enriched and H3K27me3 is reduced at a subset of plasticity genes after early-life exercise.}

The power of the Emx1-NuTRAP mouse model coupled with the simultaneous isolation procedure is the ability to directly correlate differential gene expression with candidate epigenetic mechanisms in the same cells. Hippocampal nuclei were obtained using INTACT methods in our simultaneous isolation procedure, followed by CUT\&RUN-seq from nuclear chromatin. We investigated ELE-induced changes in histone modifications H3K27me3 and H4K8ac across the genome. H4K8ac is a permissive histone modification that has been shown to be enriched at $B d n f$ and its presence correlates improved hippocampal memory after exercise [22]. H3K27me3 is a repressive histone mark and a common control in CUT\&RUN studies $[35,56]$ and is decreased at the $B d n f$ promoter region after conditional fear context training [57]. Peaks were called using SEACR [58] and histone modification enrichment peaks were overlapped with differentially expressed genes (Figure 4A). We correlated H4K8ac and H3K27me3 peaks present with upregulated and downregulated RNA as a result of ELE. The loss of either H3K27me3 or H4K8ac as a result of ELE did not correlate with changes in RNA expression. This may indicate that ELE-induced additions of these two histone modification, but not their removal, underlie differential gene expression. 93 upregulated genes correlate with new H4K8ac peaks while 33 downregulated genes correlate with new H3K27me3 peaks (Figure 4B and Supplemental Table 5). However, loss of only one H4K8ac peak correlates with downregulation of RNA, and loss of only two H3k27me3 peaks correlate with upregulation of RNA (Figure 4B and Supplemental Table 5). Unsurprisingly, many of the peaks we discovered newly present as a result of ELE did not correlate with expression changes in a large number of genes. Notable upregulated genes with new H4K8ac after ELE are Prox1, Sntb2, and Kptn. PROX1 is involved governing differentiation, maturation, and DG versus CA3 cell identity in intermediate progenitor cells of the hippocampal DG [59], while SNTB2 is involved in G protein-coupled receptor cell signaling [60]. KPTN encodes a protein involved in cytoskeletal cell structure, and its mutation can cause neurodevelopmental disability and seizures [61]. Interestingly, downregulated 
genes found to have new H3K27me3 after ELE included Col3a1, Efnb2, Epop, and Myoc. COL3A1 and MYOC are structural proteins involved in the extracellular matrix and the cytoskeleton respectively [62-64]. EFNB2 is a signaling molecule involved in cell migration and its haploinsufficiency can cause neurodevelopmental disability [65-67]. EPOP is a known editor of the chromatin landscape by altering H2Bub and H3K4me3 distributions $[68,69]$. Interestingly, many of the histone modification changes that occurred as a result of exercise appear to be in distal intergenic regions for H3K27me3 and intronic regions for H4K8ac (Figure 4C). To investigate how ELE-induced changes to histone PTM distribution might alter gene expression, we looked at the distribution of peaks around one representative gene of interest increased after ELE: Prox1 (Figure 4D). Taken together, these results suggest that ELE promotes the new addition of H4K8ac and H3K27me3 PTMs to regulate gene expression. Given that the majority of identified peaks did not correlate with DEGs, they may instead implicate regions of interest that are "primed" for facilitated gene expression following future stimuli, such as learning. 
bioRxiv preprint doi: https://doi.org/10.1101/2021.12.23.473936; this version posted December 23, 2021. The copyright holder for this preprint

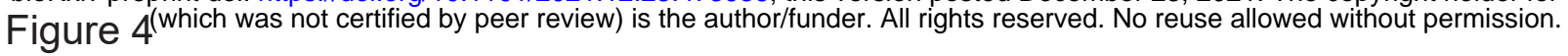
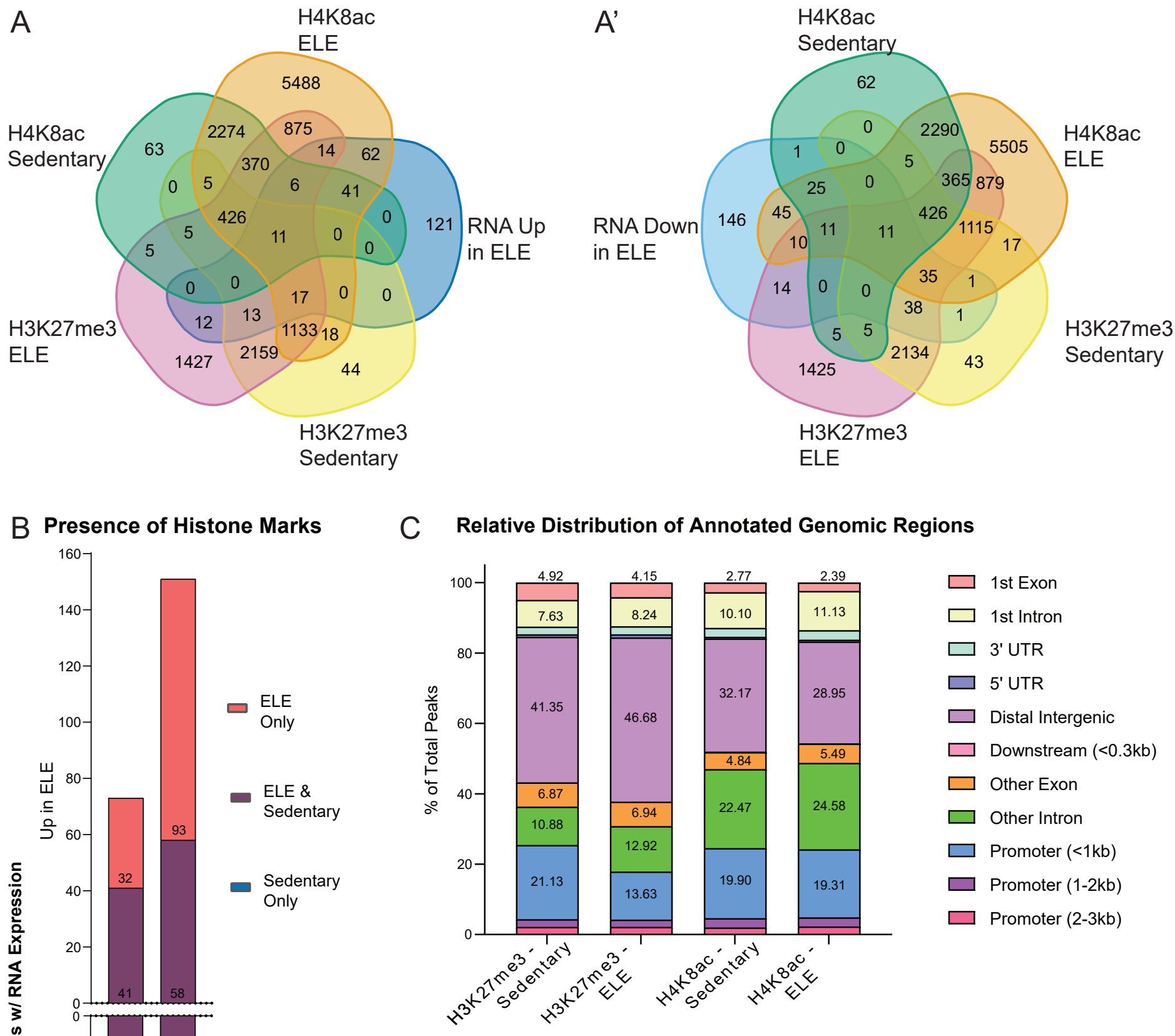

D

PROX1 Gene Tracks: chr1:189,900,750-190,843,610

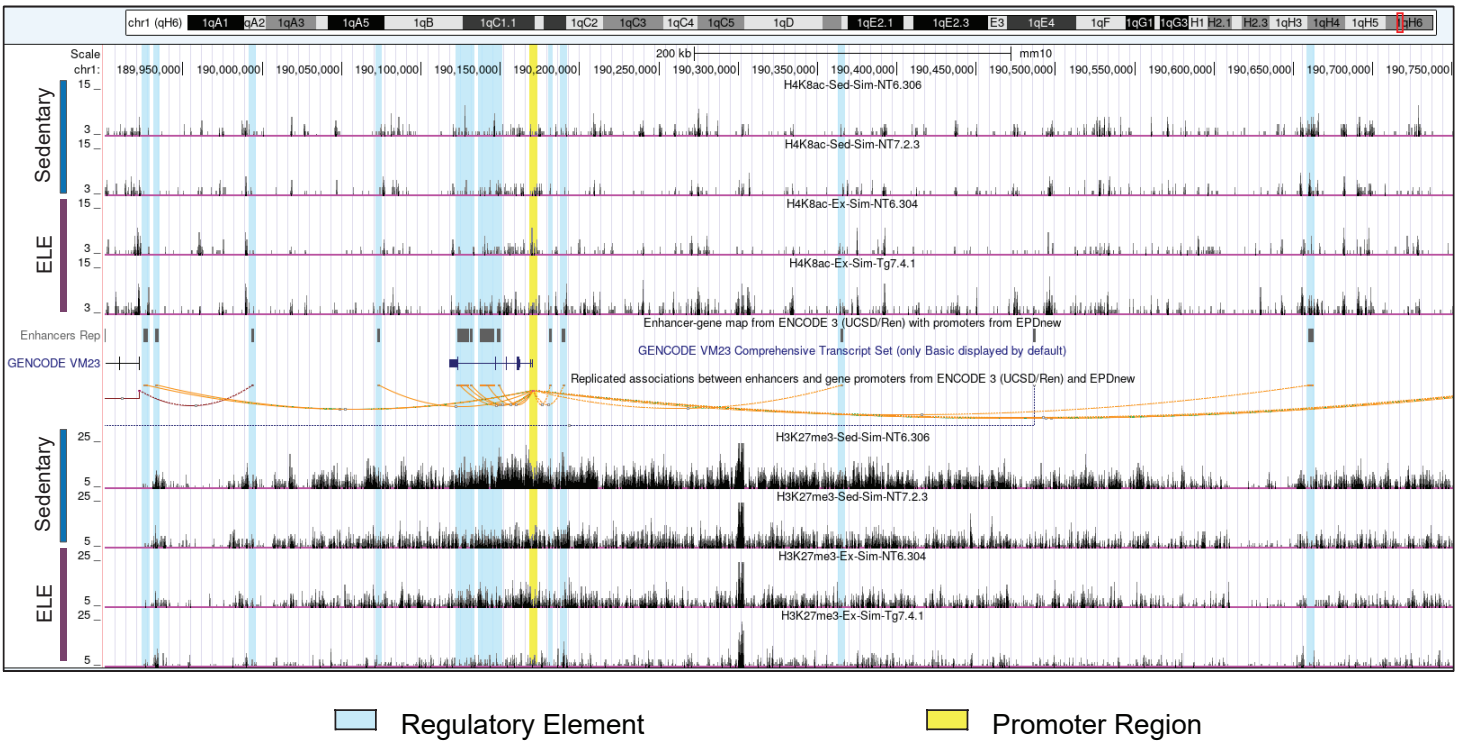


Figure 4: ELE alters H4K8ac and H3K27me3 occupancy. (A) Venn diagram of gene peaks identified in CUT\&RUN for H4K8ac and H3K27me3 by SEACR (top $1 \%$ of peaks FDR $<0.1)$ overlapped with genes upregulated (A) and downregulated (A') in RNA by ELE ( $n=2$ sedentary mice and $n=3$ ELE mice). (B) CUT\&RUN SEACR peak calls at genes that overlap with genes differentially expressed by ELE as determined by RNA-seq. (C) Stacked bar of the relative distribution of peak calls at various genomic regions identified by CUT\&RUN called using SEACR. (D) Representative track of CUT\&RUN data around Proxl showing differential peak height between ELE and sedentary conditions for H4K8ac and H3K27me3. 


\section{Neuron-specific gene expression and histone PTMs are functionally comparable between left and right hippocampal hemispheres.}

Many studies take advantage of the brain's structural symmetry by using tissue from each hemisphere for separate molecular processing. Prior evidence that hippocampal lateralization can influence LTP, hemisphere-specific glutamate receptor density, and performance in certain memory tasks [70]. We wanted to determine if there were differences in transcriptional programs resulting from ELE in left vs right hippocampi. Using the separate isolation approach described above, we compared the differential gene expression as a result of ELE between left and right hemispheres. We found that although individual gene expression patterns were different between left and right hippocampal hemispheres (Figure 5A-B), the Panther GO: Biological Processes terms were substantially similar with most categories overlapping (Figure 5C-F). This could suggest spatial or hemispheric assignment of genes with functional similarity.

Finally, we wanted to determine if left and right hippocampal hemispheres had significant differences in DEGs and CUT\&RUN sequencing peak distributions at baseline (without exercise). We performed Spearman's Rank-Order Correlation on the transcript counts from the RNA-seq $\left(R=1, p<2.2 \times 10^{-16}\right.$; Figure $\left.5 G\right)$, and the CUT\&RUN 100 bp binned peak counts for H3K27me3 (R=0.70, p<2.2x10-16; Figure $5 \mathrm{H})$ and for H4K8ac $\left(\mathrm{R}=0.59, \mathrm{p}<2.2 \times 10^{-16}\right.$; Figure $\left.5 \mathrm{I}\right)$. We found that the seq peaks and transcript counts were very similar with significance considered as $R$ values greater than 0.5 and $\mathrm{p}$ value less than $2.2 \times 10^{-16}$ (Figure $5 \mathrm{G}-\mathrm{I}$ ). These data suggest that in the sedentary condition, left and right hippocampal hemispheres yield similar normalized sequencing counts. 
bioRxiv preprint doi: https://doi.org/10.1101/2021.12.23.473936; this version posted December 23, 2021. The copyright holder for this preprint Figure $5^{\text {(which was not certified by peer review) is the author/funder. All rights reserved. No reuse allowed without permission. }}$

A

RNA Expression - Upregulated after ELE

B

RNA Expression - Downregulated after ELE

Left Hemisphere

Right Hemisphere

Left Hemisphere

Right Hemisphere
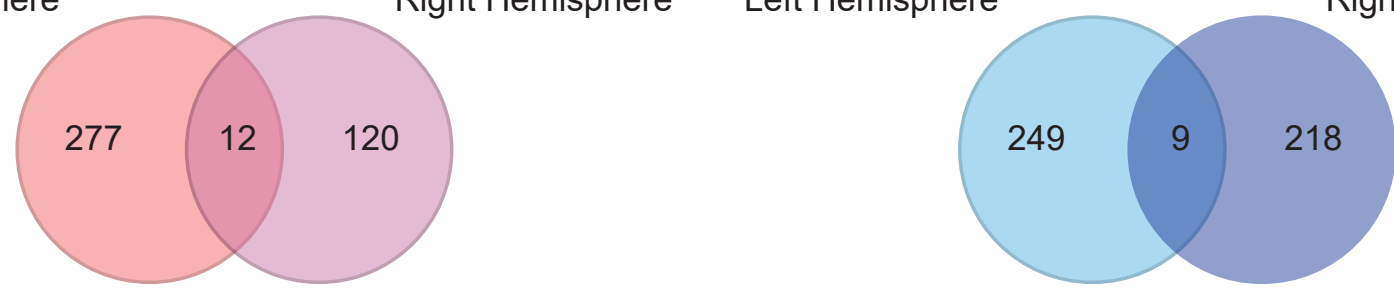

C

GO: Biological Processes - Upregulated after ELE

Right Hemisphere

D GO: Biological Processes - Downregulated after ELE Left Hemisphere

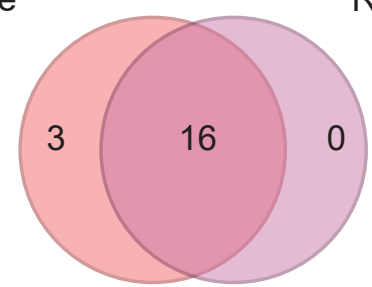
Left Hemisphere

Right Hemisphere

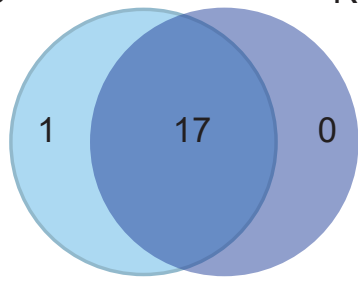

\section{E}

GO: Biological Processes Terms - Upregulated after ELE

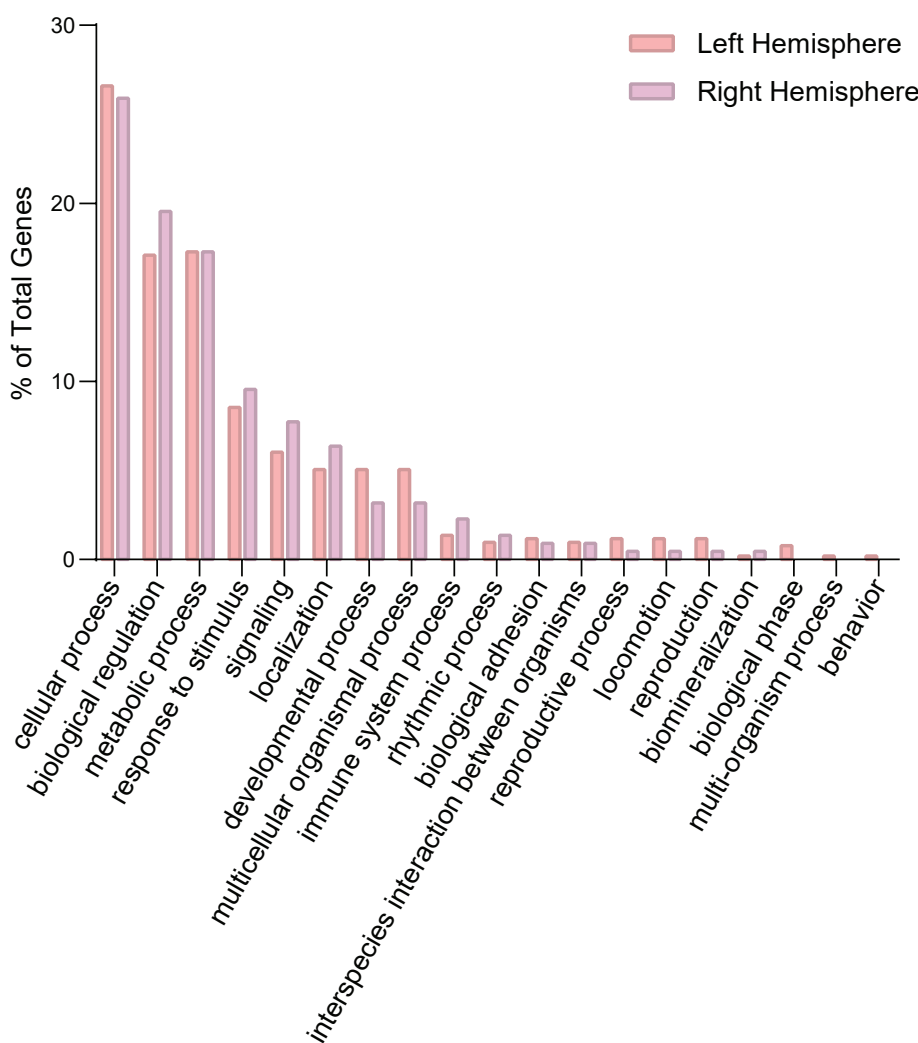

F GO: Biological Processes Terms - Downregulated after ELE

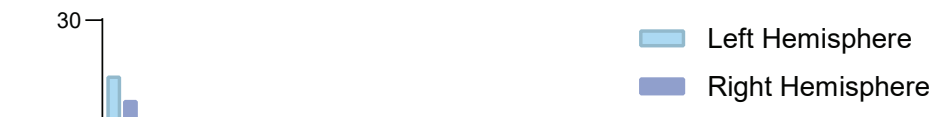

G Spearman Correlation: RNA Expression

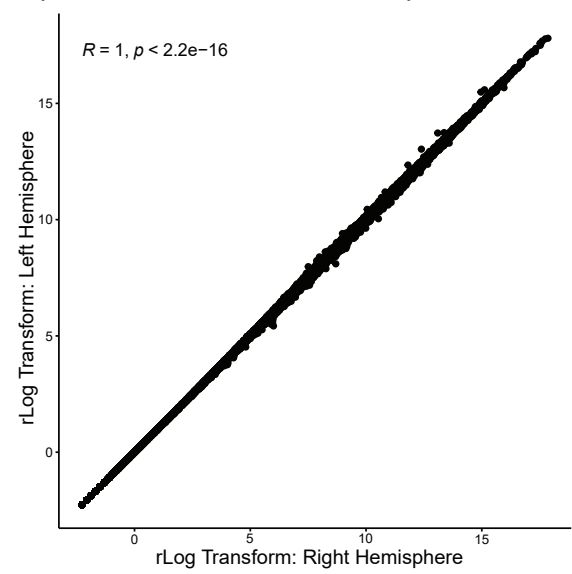

$\mathrm{H}_{\mathrm{s}}$

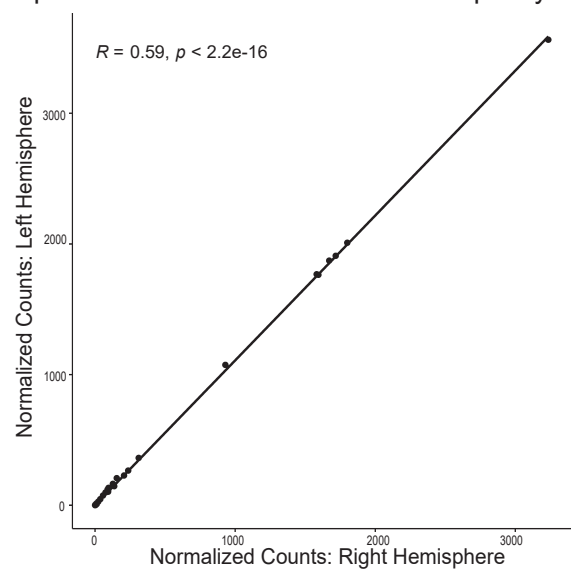

Spearman Correlation: H3K27me3 Occupancy

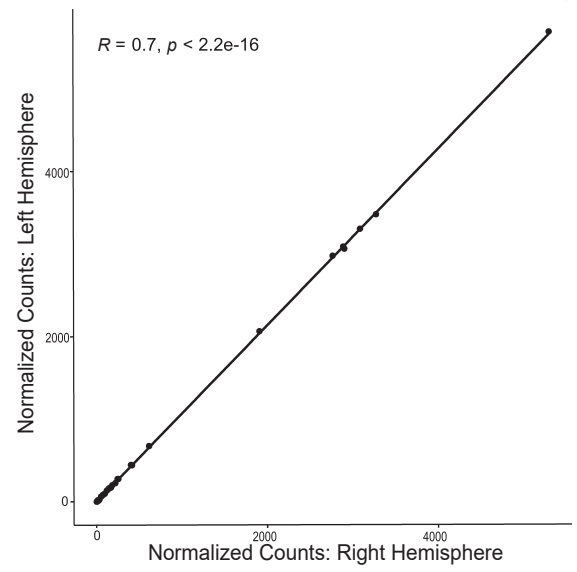




\section{Figure 5: Histone modifications and functional categorizations of gene expression are} similar regardless of hemispheric origin of hippocampal tissue. (A-B) Venn diagram of genes upregulated (A) or downregulated (B) by ELE identified between the left and right hemispheres by the separate isolation protocol RNA-seq ( $n=3$ mice per group). (C-D) Venn diagram of Panther Gene Ontology: Biological Process terms for genes upregulated (C) or downregulated (D) by ELE identified between the left and right hemispheres by the separate isolation protocol RNA-seq. (E-F) Percent of genes fitting into each GO category for the left and right hemisphere for genes upregulated (E) and downregulated (F) by ELE by separate isolation RNA-seq. (G) Spearman correlation between the left and right hemispheres RNA-seq data from the separate isolation protocol of sedentary rlog normalized expression $\left(\mathrm{R}=1, \mathrm{p}<2.2 \times 10^{-16}\right)$. (H) Spearman correlation between hemispheres for CUT\&RUN H4K8ac normalized count data for chromosome 2 in $100 \mathrm{bp}$ bins $(\mathrm{R}=0.59$, $\left.\mathrm{p}<2.2 \times 10^{-16}\right)$. (I) Spearman correlation between hemispheres for CUT\&RUN H3K27me3 normalized count data for chromosome 2 in $100 \mathrm{bp}$ bins $\left(\mathrm{R}=0.7, \mathrm{p}<2.2 \times 10^{-16}\right)$. 


\section{Discussion}

Here we present results demonstrating cell-type specific, simultaneous characterization of translating mRNA and associated histone modifications from a single population of hippocampal neurons obtained from ELE mice. To our knowledge this is the first report describing use of the NuTRAP construct in a predominantly neuronal population. Additionally, our unique experimental approach is a technical advance for NuTRAP applications: by modifying the nuclear chromatin isolation (INTACT) procedure to include cycloheximide, one can obtain both polyribosome-bound mRNA (using TRAP) and nuclear DNA from a single lysate to directly correlate gene expression programs with epigenetic regulatory mechanisms in the same set of cells. The field of neuroepigenetics has been challenged by very limited approaches for coupling cell-type specific transcriptional profiles with chromatin modifications without compromising cell integrity (through flow sorting) or genomic coverage (through single-cell sequencing). Therefore, the NuTRAP model offers an exciting opportunity to overcome these challenges and perform paired transcriptomic and epigenomic analyses in any cell type of interest for which a Cre-line exists.

Our study focuses on the impact of exercise on hippocampal gene expression and associated epigenetic modifications specifically during juvenile - adolescent periods (the fourth through sixth postnatal weeks of life in mice). We discover gene expression programs, and alterations in the histone modifications H4K8ac and H3K27me3, that may be unique to this early-life period. There have been recently published reports investigating the effects of voluntary exercise on adolescent and adult hippocampal gene expression and epigenetic modifications through RNA and DNA sequencing methods, however these used heterogenous cell populations [19, 20]. In our study, several genes with either chromatin-modifying or neurogenesisregulating properties that were either upregulated with new H4K8ac (Prox1, Sntb2, and Kptn), or downregulated with new H3K27me3 (Col3a1, Efnb2, Epop, and Myoc). For example, ELE could promote the expression of Prox1 by the addition of H4K8ac, and in turn, Prox1 can promote neurogenic fate and cell location assignment (such as DG granule cell fate over CA3 pyramidal cell identity [71]). ELE was also found to 
alter the expression of Kptn, a gene encoding a protein involved in actin dynamics and neuromorphogenesis. Increased Kptn expression and accessibility may possibly facilitate cytoskeletal changes occurring with altered synaptic plasticity after exercise. Overall, the differentially expressed genes and their associated chromatin states identified in this study provide molecular candidates involved in the effects of ELE on hippocampal function.

Traditional epigenetic and gene expression analyses of bulk hippocampal tissue are confounded by the cellular heterogeneity of the samples. Further, current methods for paired analysis of the epigenome and transcriptome are restricted by the need for multiple transgenic animal models or the use of highly expensive single-cell sequencing technologies. However, it is critical to obtain epigenomic information in a cell-type specific manner because there are unique epigenetic modifications distinguishing cell subtypes of the central nervous system (CNS) [10, 72]. Roh et al. first developed the NuTRAP mouse to characterize adipocyte-specific and hepatocyte-specific epigenetic and transcriptional profiles [29]. The NuTRAP mouse has since been used to perform paired transcriptome and DNA modification analysis in astrocytes and microglia, demonstrating its utility in CNS cell-types [38]. The Emx1-NuTRAP mouse described here provides rapid, cost-effective characterization of neuron-specific changes to epigenetic state and gene expression using whole-tissue homogenates with heterogeneous cell types. In our technical validation experiments, the combined INTACT and TRAP isolation procedures (simultaneous isolation) produced high quality RNA and DNA of sufficient concentration for downstream sequencing studies from a single mouse hippocampus. This method effectively increases the total sample size used for each analysis as the tissue sample does not need to be separated for DNA and RNA isolation. CUT\&RUN and CUT\&Tag allow for low-input cell-specific chromatin analysis $[58,73]$ further reducing the required amount of starting material. Indeed, the DNA obtained from the simultaneous INTACT and TRAP isolations could be used for other applications for evaluating the chromatin landscape, including ATAC-seq.

A common approach to paired transcriptomic and epigenomic analyses is to use separate brain tissue from one hemisphere for gene expression studies and the 
other side for epigenetic analyses [74]. A previous study utilizing the NuTRAP allele in non-neuronal brain cells used separate brain hemispheres to perform INTACT and TRAP [75]. However, this assumes that cells of the same type from each half of the tissue will show the same epigenetic and transcriptional profile. This is not necessarily the case for all cell types, especially in the CNS, as structures of the brain can show high degrees of hemispheric specialization [76-80]. To address the degree of transcriptional or epigenetic difference between hippocampi from opposite hemispheres, we performed separate INTACT and TRAP isolations using hippocampal tissue from one hemisphere for DNA isolation and the contralateral hippocampus for mRNA isolation. We found that although differential gene expression and histone modifications differed between left and right hippocampi, functional analysis using gene ontology revealed similar biological processes between the two sides.

In summary, our work reveals ELE-induced transcriptomic and epigenomic signatures in hippocampal neurons using the Emx1-NuTRAP mouse we developed for such comparisons. Presently, the field is shifting to single-cell resolution for sequencing studies, however our study was performed in bulk tissue enriched for neurons. As the goal of this work was to characterize the transcriptional and epigenomic effects of early life exercise, cell subtype identity (as is often a priority in single-cell approaches) was not necessary to address this study's questions. Furthermore, single-cell approaches bias against lowly-expressed genes, which presents the risk of not capturing small expression changes in rate-limiting genes that may have large changes in function as a result of ELE. A limiting factor for the use of the NuTRAP transgenic mouse is the requirement of a Cre-line to exist for the experimenter's cell type of interest. In our Emx1-NuTRAP model the the Crerecombinase in Emx1 expressing cells is constitutively expressed, and this may present off-target effects when present during development. Despite this, our study uses a feasible, previously undescribed approach in the field of neuroepigenetics to pair transcriptomic and epigenomic bulk sequencing results from a population of hippocampal neurons, revealing new molecular epigenetic candidates for further 
bioRxiv preprint doi: https://doi.org/10.1101/2021.12.23.473936; this version posted December 23, 2021. The copyright holder for this preprint (which was not certified by peer review) is the author/funder. All rights reserved. No reuse allowed without permission.

mechanistic studies to examine the effects of early life exercise on hippocampal function. 


\section{Methods}

\section{Resource Availability}

\section{Lead Contact}

Further information and requests for resources and reagents should be directed to and will be fulfilled by the lead contact, Dr. Autumn Ivy (aivy@uci.edu)

\section{Materials Availability}

- No new reagents or materials were generated for this study.

- The mouse lines used for this study are available from Jackson Labs (Emx1IRES-Cre knock-in mice, Jackson Laboratory Stock No: 005628, and NuTRAP mice, Jackson Laboratory Stock No: 029899).

\section{Data Availability}

RNA seq and CUT\&RUN seq data will be deposited and publicly available as of the date of publication. Accession numbers will be listed here pending publication. Any additional information required to reanalyze the data reported in this paper is available from the lead contact upon request. Supplemental tables will be available upon publication.

\section{Animals}

Emx1-IRES-Cre knock-in mice (Jackson Laboratory Stock No: 005628) and NuTRAP mice (Jackson Laboratory Stock No: 029899) were obtained from Jackson Laboratories. Female Emx1-IRES-Cre and male NuTRAP mice were crossed to generate 
the Emx1-NuTRAP mice used for this study. Mice were given free access to food and water and lights were maintained on a standard 12-hour light/dark cycle. On postnatal day (P) 21, mice were weaned and pair-housed in either standard bedding cages or cages equipped with a running wheel. Only male mice were used for the studies performed. Experiments were conducted according to US National Institutes of Health guidelines for animal care and use and were approved by the Institutional Animal Care and Use Committee of the University of California, Irvine.

\section{Exercise Paradigm}

After weaning on P21, male mice from the same litters were randomly divided and pair-housed in either standard cages without a wheel or cages equipped with a stainless-steel running wheel similar to methods already described [17]. Animals housed with running wheels had free access to the wheels from P21-41. Real-time data acquisition (Vital View Software) was used to track distance ran recorded by magnetic sensors that detect wheel revolutions. Each monitored wheel tracked running distance for the entire cage (two mice per cage). Revolutions were quantified for every minute daily for the duration of the exercise period and converted to distance ran per cage $(\mathrm{km})$.

\section{Simultaneous RNA and Nuclear Isolation}

On P42 both ELE $(n=4)$ and sedentarycontrol $(n=4)$ mice were sacrificed by rapid cervical dislocation and both hemispheres of the hippocampus were dissected out and collected for tissue processing. Hippocampal tissue samples were mechanically homogenized in $1 \mathrm{~mL}$ of nuclear preparation buffer (NPB) (10mM HEPES (pH7.5); 
1.5mM MgCl2; 10mM KCl; 250mM sucrose; 0.1\% IGEPAL CA-630; 0.2mM DTT;

$100 \mu \mathrm{g} / \mathrm{mL}$ cycloheximide; 1x Complete EDTA-free protease inhibitor (Roche)). by razor blade. Samples were then triturated with an $18 \mathrm{G}$ needle and syringe 50x on ice.

Homogenized tissue samples were pipetted slowly onto $100 \mu \mathrm{m}$ cell strainer caps on $5 \mathrm{~mL}$ tubes and centrifuged at $1,000 \mathrm{rcf}$ for 10 minutes at $4^{\circ} \mathrm{C}$. Cell pellets were resuspended and samples were pipetted onto $40 \mu \mathrm{m}$ cell strainer caps on $5 \mathrm{~mL}$ tubes and centrifuged at $1,000 \mathrm{rcf}$ for 10 minutes at $4^{\circ} \mathrm{C}$. For nuclear isolation, $100 \mu \mathrm{L}$ of

Streptavidin-coated Dynabeads were washed twice with $1 \mathrm{~mL}$ NPB and resuspended with $100 \mu \mathrm{L}$ NPB. Cell pellets were resuspended and added to washed Streptavidin-coated Dynabeads. Samples were briefly pipetted to thoroughly suspend the beads and incubated on ice for 20 minutes. Samples were placed on a magnetic stand to pull down nuclei bound to the beads. The supernatant from each sample was slowly pipetted off the beads and transferred to new $1.5 \mathrm{~mL}$ tubes for RNA isolation (described later). Nuclei-bound beads were washed twice with 1mL INTACT buffer (10mM HEPES (pH7.5); 1.5mM $\mathrm{MgCl} 2 ; 10 \mathrm{mM} \mathrm{KCl} ; 250 \mathrm{mM}$ sucrose; 0.1\% IGEPAL CA-630; 1x Complete EDTA-free protease inhibitor (Roche)) and resuspended in 100 $\mu$ L INTACT buffer. Bead-bound nuclei were flash frozen on dry ice and stored at $-80^{\circ} \mathrm{C}$ until ready for CUT\&RUN chromatin digestion and DNA purification.

For RNA isolation, $100 \mu \mathrm{L}$ of concentrated TRAP isolation buffer (10mM HEPES pH 7.5; 117mM MgCl2; $1 \mathrm{M} \mathrm{KCl} ; 10 \%$ IGEPAL CA-630; 100 $\mu \mathrm{g} / \mathrm{mL}$ cycloheximide; $11 \mathrm{mg} / \mathrm{mL}$ sodium heparin; 20mM DTT; 2.2 units/ $\mu \mathrm{L}$ Rnasin; 1x Complete EDTA-free protease inhibitor (Roche)) was added to each sample (suppernatant above). Samples were mixed by gentle pipetting and centrifuged at $16,000 \mathrm{rcf}$ for 10 minutes at $4^{\circ} \mathrm{C}$. The 
supernatant from each sample was transferred to new $1.5 \mathrm{~mL}$ tubes and $50 \mu \mathrm{L}$ of protein-G Dynabeads was added to each sample. (To bind anti-GFP, $100 \mu \mathrm{L}$ of protein-G Dynabeads were washed twice with $500 \mu \mathrm{L}$ NPB and resuspended to a final $100 \mu \mathrm{L}$ volume. $2 \mu \mathrm{L}$ anti-GFP antibody (Sigma-Aldrich, G6539, Lot:128M4867V) was added to each tube and incubated for 1 hour on a rotating nutator at $4^{\circ} \mathrm{C}$. Excess anti-GFP was washed off with $200 \mu \mathrm{L}$ NPB and beads were resuspended in a final $100 \mu \mathrm{L}$ volume.) Samples were incubated for $3 \mathrm{~h}$ on a rotating nutator at $4^{\circ} \mathrm{C}$. Beads were washed $3 \mathrm{x}$ in $1 \mathrm{~mL}$ high salt wash buffer (HSWB) (50mM Tris $\mathrm{pH} 7.5 ; 12 \mathrm{mM} \mathrm{MgCl} 2 ; 300 \mathrm{mM} \mathrm{KCl}$; 1\% IGEPAL CA-630; $2 \mathrm{mM}$ DTT; $100 \mathrm{ug} / \mathrm{mL}$ cycloheximide) at $4^{\circ} \mathrm{C}$. After the third wash the beads were slowly resuspended in $350 \mu \mathrm{L}$ RLT buffer (from Qiagen RNeasy Mini kit \#74004) by slowly pipetting up and down and incubated on a rotating nutator for 30 minutes at room temperature. Samples were placed on a magnetic stand to pull down the beads, and the supernatant from each were transferred to new $1.5 \mathrm{~mL}$ tubes and stored at $-80^{\circ} \mathrm{C}$ until ready for RNA purification.

\section{Separate TRAP RNA Isolation}

On P42, mice ( $\mathrm{n}=3$ ) were sacrificed and the hippocampus was dissected out as described above. Either the left or right brain hemisphere of the hippocampus was processed for TRAP RNA isolation using methods similar to those previously described $[28,81]$ and the other hemisphere was processed for INTACT nuclear isolation (see below). Hippocampal tissue was homogenized with a motorized pestle in $1 \mathrm{ml}$ of TRAP homogenization buffer (50mM Tris, pH 7.5; $12 \mathrm{mM} \mathrm{MgCl}_{2} ; 100 \mathrm{mM} \mathrm{KCl} ; 1 \% \mathrm{NP}-40$; $100 \mu \mathrm{g} / \mathrm{ml}$ cycloheximide; $1 \mathrm{mg} / \mathrm{ml}$ sodium heparin; $2 \mathrm{mM}$ DTT; 0.2 units/ $\mu 1$ RNasin; $1 \mathrm{x}$ 
Complete EDTA-free protease inhibitor (Roche)). Samples were centrifuged at 16,000rcf for 10 minutes at $4^{\circ} \mathrm{C}$. The supernatant from each sample was collected and incubated with $50 \mu \mathrm{L}$ of protein G Dynabeads (washed twice in TRAP homogenization buffer without RNasin or protease inhibitor). Samples were incubated for 30 minutes on a rotating nutator at $4^{\circ} \mathrm{C}$. Following the incubation, $100 \mu \mathrm{L}$ of anti-GFP bound protein-G Dynabeads was added to each sample. (To bind anti-GFP, $100 \mu \mathrm{L}$ of protein-G Dynabeads were washed twice with $500 \mu \mathrm{L}$ TRAP homogenization buffer without RNasin or protease inhibitor and resuspended to a final $100 \mu \mathrm{L}$ volume. $2 \mu \mathrm{L}$ anti-GFP antibody (Sigma-Aldrich, G6539, Lot:128M4867V) was added to each tube and incubated for 1 hour on a rotating nutator at $4^{\circ} \mathrm{C}$. Excess anti-GFP was washed off with $200 \mu \mathrm{L}$ TRAP homogenization buffer without RNasin or protease inhibitor and beads were resuspended in a final $100 \mu \mathrm{L}$ volume.) Samples were incubated for 3 hours on a rotating nutator at $4^{\circ} \mathrm{C}$. Beads were washed $3 \mathrm{x}$ in $1 \mathrm{~mL}$ HSWB at $4^{\circ} \mathrm{C}$. After the final wash, the beads were resuspended in 350 $\mu$ L RLT buffer (from Qiagen RNeasy Mini kit \#74004) and incubated on a rotating nutator for 30 minutes at room temperature. Following the incubation, samples were transferred to new $1.5 \mathrm{~mL}$ tubes and stored at $-80^{\circ} \mathrm{C}$ until ready for RNA purification.

\section{Separate INTACT Nuclear Isolation}

The remaining hippocampal hemisphere from each mouse (not used for TRAP RNA isolation above) was processed for INTACT DNA isolation using methods similar to those described $[28,82]$. Tissue samples were quickly chopped with a razor blade, added to $1 \mathrm{~mL}$ of INTACT buffer, and triturated with an $18 \mathrm{G}$ needle and syringe 50x on 
ice. Samples were then added onto $100 \mu \mathrm{m}$ cell strainer caps on $5 \mathrm{~mL}$ tubes and centrifuged at $1,000 \mathrm{rcf}$ for 10 minutes at $4^{\circ} \mathrm{C}$. Cell pellets were resuspended and samples were pipetted onto $40 \mu \mathrm{m}$ cell strainer caps and centrifuged at 1,000rcf for 10 minutes at $4^{\circ} \mathrm{C} .100 \mu \mathrm{L}$ of streptavidin coated Dynabeads (washed twice with $1 \mathrm{~mL}$ INTACT buffer) were added to each sample, and samples were incubated on ice for 20 minutes. After incubation, nuclei bound beads were washed twice with $1 \mathrm{~mL}$ and resuspended in $100 \mu \mathrm{L}$ INTACT buffer. Bead-bound nuclei were flash frozen on dry ice and stored at $-80^{\circ} \mathrm{C}$ until ready for CUT\&RUN chromatin digestion and DNA purification.

\section{RNA Purification and Sequencing Library Preparation}

RNA was purified using the Qiagen RNeasy kit according to the manufacturer's protocol. After purification, RNA quantity and quality were checked using the Qubit RNA High Sensitivity assay and Agilent Bioanalyzer's Eukaryote Total RNA Pico assay, respectively, at the University of California, Irvine Genomics High Throughput Facility (UCI GHTF). Using an input of 15ng of total RNA, mRNA was isolated using NEXTFLEX Poly(A) Beads 2.0, and the mRNA sequencing libraries were generated using the NEXTFLEX Rapid Directional RNA-Seq Kit 2.0 according to the manufacturer's instructions. Final libraries were sent to the UCI GHTF for Qubit dsDNA High Sensitivity assay and the Agilent Bioanalyzer DNA High Sensitivity assay to determine the quantity and quality of the RNA-seq libraries, respectively. Libraries were sequenced at the UCI GHTF using 100bp paired end reads on a single lane of an Illumina NovaSeq6000 to a minimum sequencing depth of 50 million reads. 


\section{Cleavage Under Target and Release Using Nuclease (CUT\&RUN)}

For chromatin analysis, CUT\&RUN was performed using the EpiCypher CUTANA CUT\&RUN protocol with slight modifications similar to what has already been described [35]. Nuclei bound to beads were thawed and pulled down on a magnetic stand. The supernatant was removed and beads were resuspended in $200 \mu \mathrm{L}$ wash buffer (20mM HEPES pH 7.5; $150 \mathrm{mM} \mathrm{NaCl} ; 0.5 \mathrm{mM}$ spermidine; $1 \mathrm{x}$ Complete EDTA-free protease inhibitor (Roche)). Each sample was divided into three aliquots $(50 \mu \mathrm{L}$ for anti-H3K27me3 (Cell Signaling Technologies, C36B11, Lot: 16), $100 \mu \mathrm{L}$ for anti-H4K8ac (Epicypher, 13-0047, Lot: 20202001-11), and $50 \mu \mathrm{L}$ for anti-IgG control (Rabbit IgG Fisher Scientific, 026102)). Beads were pulled down on a magnetic stand, resuspended in $100 \mu \mathrm{L}$ wash buffer and incubated for 10 minutes at room temperature. After incubation the beads were pulled down, the supernatant was removed, and the beads were resuspended in $50 \mu \mathrm{L}$ ice cold antibody buffer (20mM HEPES pH 7.5; 150mM NaCl; 0.5mM spermidine; 0.001mM digitonin; 2mM EDTA; 1x Complete EDTA-free protease inhibitor (Roche)). $0.5 \mu \mathrm{L}$ of the appropriate antibody was added to each sample, and the samples were mixed by gentle pipetting prior to overnight incubation at $4^{\circ} \mathrm{C}$ on a nutator.

On the second day, the beads were pulled down on a magnetic stand, the supernatant was removed and the beads were washed twice in $250 \mu \mathrm{L}$ ice cold wash buffer with $0.001 \%$ digitonin. After the washes, beads were resuspended in $50 \mu \mathrm{L}$ ice cold wash buffer with $0.001 \%$ digitonin, and $2.5 \mu \mathrm{L}$ of CUTANA pAG-MNase was added to each sample and mixed by gentle pipetting. Samples were incubated for 10 minutes at room temperature and beads were pulled down on a magnetic stand. The supernatant was 
removed, and the beads were washed twice in $250 \mu \mathrm{L}$ ice cold wash buffer with $0.001 \%$ digitonin and resuspended in $50 \mu \mathrm{L}$ of the same. $1 \mu \mathrm{L}$ of $100 \mathrm{mM} \mathrm{CaCl}_{2}$ was added to each sample and mixed by gentle pipetting. Samples were incubated for 2 hours on a nutator at $4^{\circ} \mathrm{C}$. Following the incubation, $33 \mu \mathrm{L}$ of stop buffer $(340 \mathrm{mM} \mathrm{NaCl} ; 20 \mathrm{mM}$ EDTA; $4 \mathrm{mM}$ EGTA; $50 \mu \mathrm{g} / \mathrm{mL}$ RNase A; $50 \mu \mathrm{g} / \mathrm{mL}$ Glycogen) and $1.65 \mu \mathrm{L}$ of Spike-In DNA (Cell Signaling Technology, \#40366) was added to each sample. Samples were incubated in a preheated thermocycler for 10 minutes at $37^{\circ} \mathrm{C}$. After incubation, samples were transferred to new $1.5 \mathrm{~mL}$ tubes and immediately processed for DNA purification.

\section{DNA Purification and Library Preparation}

DNA was purified using the Monarch PCR and DNA cleanup kit (New England Biolabs, 13-0041) according to the manufacturer's instructions. After purification, DNA fragments were used to generate sequencing libraries using the NEXTFLEX Rapid DNA-Seq Kit 2.0 according to the manufacturer's instructions. Indices were diluted to a final concentration of 1:1,000 before being added to samples, and a total of 13 PCR cycles was used for DNA amplification of the libraries. Final libraries were sent to the UCI GHTF for Qubit dsDNA High Sensitivity assay and the Agilent Bioanalyzer DNA High Sensitivity assay to determine the quantity and quality of the CUT\&RUN-seq libraries, respectively. Libraries were sequenced at the UCI GHTF using 100bp paired end reads on a single lane of an Illumina NovaSeq6000 to a minimum sequencing depth of 10 million reads. 
cDNA was generated from TRAP-isolated and total input RNA samples using the Transcriptor First Strand cDNA Synthesis Kit (Roche) following the manufacturer's instructions. Relative gene expression for Aqp4 (F: 5'-ATCCAGCTCGATCTTTTGGA 3', R: 5'-TGAGCTCCACATCAGGACAG -3'), Tubb3 (F: 5'-

GTCTCTAGCCGCGTGAAGTC -3', R: 5'-GCAGGTCTGAGTCCCCTACA -3'), Cd11b (F: 5'-CCCATGACCTTCCAAGAGAA -3', R: 5'-

ACACTGGTAGAGGGCACCTG -3'), and $\operatorname{Mog}\left(\mathrm{F}: 5^{\prime}\right.$ AAGAGGCAGCAATGGAGTTG -3', R: 5'-GACCTGCAGGAGGATCGTAG -3') was determined by qPCR using FastStart Essential DNA Green Master Mix (Roche, 06402712001) following the manufacturer's protocol. Cycle counts for mRNA quantification were normalized to Gapdh (F: 5'-CGTCCCGTAGACAAAATGGT -3', R: 5'-GAATTTGCCGTGAGTGGAGT -3'). Quantification was performed using the Pfaffl method [83].

\section{Immunofluoresence}

Emx1-NuTRAP mice were anesthetized intraperitoneally with $50 \mathrm{mg} / \mathrm{kg}$ of sodium pentobarbital and transfused transcardially with $4 \%$ paraformaldehyde (PFA) in 1x PBS. After perfusion, whole brains were dissected out and incubated in $4 \%$ PFA overnight at $4^{\circ} \mathrm{C}$. After fixation, brains were incubated in $30 \%$ sucrose for 72 hours and mounted in optimal cutting temperature (OCT) compound. $50 \mu \mathrm{m}$ coronal sections were sliced through the hippocampus using a microtome, and every tenth section was collected in $1 \mathrm{~mL}$ of cryoprotectant and stored at $-20^{\circ} \mathrm{C}$ until ready for immunofluorescent labeling. For immunofluorescence, sections were brought up to room temperature and washed $2 \mathrm{x}$ 
in $1 \mathrm{x}$ PBS on a nutator for 5 minutes each. Sections were then washed 3x in 1x PBS with $0.3 \%$ triton-X. After washes, sections were incubated with mCherry polyclonal antibody (ThermoFisher, PA5-34974, Lot: UG2804409F) at a 1:200 dilution in blocking solution (1x PBS with $1 \% \mathrm{BSA}$ ) overnight at $4^{\circ} \mathrm{C}$ on a nutator. The following day, sections were washed $3 x$ in $1 x$ PBS. Sections were incubated for two hours at room temperature with goat anti-rabbit Alexa Fluor 594 secondary antibody (Invitrogen, A-11012, Lot:

2119134) at a 1:200 dilution in blocking solution. After incubation, the sections were washed twice in 1x PBS. To label nuclei, sections were incubated with DAPI at a 1:10,000 dilution in 1x PBS for 20 minutes. Sections were then washed $3 \mathrm{x}$ in $1 \mathrm{x}$ PBS and cover slipped with Vectatshield®. Fluorescent images were acquired using a Keyence BZ-X810 All-in-One Fluorescence microscope using the optical sectioning module.

\section{Fluorescence Activated Cell Sorting}

Whole hippocampal tissue from Emx-NuTRAP mice was isolated and fluorescence activated cell sorting (FACS) using an Aria Fusion from the University of California, Irvine Stem Cell Core was performed to characterize neuronal and astrocytic NuTRAP cassette expression using methods similar to what has been described [84]. We used antibodies for THY-1(Santa Cruz Biotechnology, sc-53116 AF647, Lot: F2716; concentration: 1:50) and S100 (Abcam, ab41548, Lot:GR3326165-1; concentration: 1:200) with a AlexaFluor 405 goat anti-rabbit IgG (H+L) (Invitrogen, A31556, Lot: 2273716; concentration:1:800).

\section{Bioinformatics Analysis}


Reference assembly: For all sequencing analysis that required it, the primary reference assembly used was Genome Reference Consortium Mouse Build 38 patch release 6 (GRCm38.p6).

RNA-seq analysis: FastQ files were quality checked for sequencing errors using FastQC (version 0.11.9)[85]. No files were found to have sufficient quality errors to discount their use. Files were aligned using STAR Aligner (version 2.7.3a) [86]. Duplicate reads were removed using Picard Tools (version 1.87) [87]. SAM Tools was used to convert BAM files to SAM files for use in downstream analysis. FastQC, alignment and duplicate removal were all preformed on the High-Powered Compute Cluster (HPC3) operated by The Research Cyberinfrastructure Center (RCIC) at the University of California, Irvine (UCI). R (version 4.1.0) [88] was used for differentially expressed genes (DEG) analysis. Genomic Alignments (version 1.28.0) [89] (summarizeOverlaps mode="IntersectionNotEmpty", singleEnd=FALSE, ignore.strand=FALSE, fragments=TRUE), Genomic Features (version 1.44.0) (exons by gene), and R SAM Tools (version 2.8.0) [90] (yieldSize=100000) were used to extract a count matrix and generate a summarized experiment object. DEseq2 (version 1.32.0) [91] was used to perform a DEG analysis. Ensemble IDs were converted to gene symbols using BiomaRt (version 2.48.1) [92, 93]. PCA plots using the top 2000 genes were generated using ggplot2 (version 3.3.4) [94]. Samples were determined as outliers if a PCA plot that included them showed that most of the difference between the samples was represented by those samples deemed outliers alone (3 samples). Heatmaps were generated using gplots (version 3.1.1) [95] and RColorBrewer (version1.1-2) [96]. Volcano Plots were generated using ggplot2 
[94]. Venn diagrams were generated using the Venn diagram tool from

Bioinformatics and Evolutionary Genomics [97]. Gene ontology analysis was performed using Panther Classification System (version 16.0) [41]. Genes were also categorized and a leading edge heatmap was also generated using Gene Set Enrichment Analysis (version GSEA 4.1.0) [52]. Upstream regulators were identified from the DEGs upregulated by ELE using Qiagen's Ingenuity Pathway Analysis (IPA) (Fall 2021 Release) [42].

CUT\&RUN-seq analysis: FastQ files were quality checked for sequencing errors using FastQC (version 0.11.9) [85]. No files were found to have sufficient quality errors to discount their use. FastQ files were aligned using Bowtie2 (version 2.4.1) [98]. Significant peaks were called from these aligned files using SEACR, developed in CUT\&Tag for efficient epigenomic profiling of small samples and single cells $[58,98]$, according to CUT\&Tag Data Processing and Analysis Tutorial (updated August 12 2020): https://www.protocols.io/view/cut-amp-tag-data-processingand-analysis-tutorial-bjk2kky. An FDR higher than 0.1 was considered too high for called peaks. This threshold excluded 4 samples. Peaks were called using the stringent and 0.01 settings. Significant peaks were annotated using ChIPseeker (version 1.8.6) [99] using TxDb.Mmusculus.UCSC.mm10.knownGene [100] as a reference with the following settings: tssRegion $=c(-3000,3000), \mathrm{T} x \mathrm{Db}=$ TxDb.Mmusculus.UCSC.mm10.knownGene, level = "transcript", assignGenomicAnnotation = TRUE, genomicAnnotationPriority = c("Promoter", "5UTR", "3UTR", "Exon", "Intron", "Downstream", "Intergenic"), annoDb = NULL, addFlankGeneInfo $=$ FALSE, flankDistance $=5000$, sameStrand $=$ FALSE, 
ignoreOverlap $=$ FALSE, ignoreUpstream $=$ FALSE, ignoreDownstream $=$ FALSE, overlap = "TSS", verbose $=$ TRUE). This approach annotated peaks to the closest gene by distance from the promoter, except if that peak falls within a gene before the distal intergenic region. Ensemble IDs were converted to gene symbols using BiomaRt $[92,93]$. Gene lists of peaks present in each condition were used to generate Venn diagrams, as with RNA-seq analysis[97]. Further Venn diagrams were generated comparing DEGs with peak calls. Peaks were visualized using UCSC Genome Browser and Genome Browser in a Box [101-103].

Normalized counts (for read depth) at binned sections of the DNA as well as RNA-seq gene expression count (normalized for read depth) were compared between simultaneous and separate isolations as well as left and right hemispheres using spearman correlations in R with the package ggpubr (version 0.4.0) [104]. Bar plots were generated using Graph Pad 9 PRISM. 


\section{References}

1. Bedrosian, T.A., et al., Early life experience drives structural variation of neural genomes in mice. Science, 2018. 359(6382): p. 1395-1399.

2. Stroud, H., et al., Early-Life Gene Expression in Neurons Modulates Lasting Epigenetic States. Cell, 2017. 171(5): p. 1151-1164.e16.

3. Herre, M. and E. Korb, The chromatin landscape of neuronal plasticity. Curr Opin Neurobiol, 2019. 59: p. 79-86.

4. Campbell, R.R. and M.A. Wood, How the epigenome integrates information and reshapes the synapse. Nat Rev Neurosci, 2019. 20(3): p. 133-147.

5. Kronman, H., et al., Long-term behavioral and cell-type-specific molecular effects of early life stress are mediated by H3K79me2 dynamics in medium spiny neurons. Nat Neurosci, 2021. 24(5): p. 667-676.

6. Sagarkar, S., et al., LSD1-BDNF activity in lateral hypothalamus-medial forebrain bundle area is essential for reward seeking behavior. Prog Neurobiol, 2021. 202: p. 102048.

7. Webb, W.M., et al., The SETD6 Methyltransferase Plays an Essential Role in Hippocampus-Dependent Memory Formation. Biological psychiatry, 2020. 87(6): p. 577-587.

8. Bolton, J.L., et al., Unexpected Transcriptional Programs Contribute to Hippocampal Memory Deficits and Neuronal Stunting after Early-Life Adversity. Cell Rep, 2020. 33(11): p. 108511.

9. Campbell, R.R., et al., HDAC3 Activity within the Nucleus Accumbens Regulates Cocaine-Induced Plasticity and Behavior in a Cell-Type-Specific Manner. The Journal of neuroscience : the official journal of the Society for Neuroscience, 2021. 41(13): p. 2814-2827.

10. Lister, R., et al., Global Epigenomic Reconfiguration During Mammalian Brain Development. Science, 2013. 341(6146): p. 1237905.

11. Lavery, L.A. and H.Y. Zoghbi, The distinct methylation landscape of maturing neurons and its role in Rett syndrome pathogenesis. Curr Opin Neurobiol, 2019. 59: p. 180-188.

12. Aguilar, R., et al., Epigenetic silencing of the osteoblast-lineage gene program during hippocampal maturation. J Cell Biochem, 2021. 122(3-4): p. 367-384.

13. West, A.E. and M.E. Greenberg, Neuronal activity-regulated gene transcription in synapse development and cognitive function. Cold Spring Harb Perspect Biol, 2011. 3(6).

14. Bale, T.L., et al., Early life programming and neurodevelopmental disorders. Biol Psychiatry, 2010. 68(4): p. 314-9.

15. Cotman, C.W., N.C. Berchtold, and L.-A. Christie, Exercise builds brain health: key roles of growth factor cascades and inflammation. Trends in Neurosciences, 2007. 30(9): p. 464-472.

16. Voss, M.W., et al., Bridging animal and human models of exercise-induced brain plasticity. Trends Cogn Sci, 2013. 17(10): p. 525-44.

17. Ivy, A.S., et al., A Unique Mouse Model of Early Life Exercise Enables Hippocampal Memory and Synaptic Plasticity. Scientific Reports, 2020. 10(1): p. 9174. 
18. Berchtold, N.C., N. Castello, and C.W. Cotman, Exercise and time-dependent benefits to learning and memory. Neuroscience, 2010. 167(3): p. 588-97.

19. Urdinguio, R.G., et al., Physical exercise shapes the mouse brain epigenome. Mol Metab, 2021. 54: p. 101398.

20. Abel, J.L. and E.F. Rissman, Running-induced epigenetic and gene expression changes in the adolescent brain. Int J Dev Neurosci, 2013. 31(6): p. 382-90.

21. Ieraci, A., et al., Physical exercise and acute restraint stress differentially modulate hippocampal brain-derived neurotrophic factor transcripts and epigenetic mechanisms in mice. Hippocampus, 2015. 25(11): p. 1380-92.

22. Intlekofer, K.A., et al., Exercise and Sodium Butyrate Transform a Subthreshold Learning Event into Long-Term Memory via a Brain-Derived Neurotrophic factor-Dependent Mechanism. Neuropsychopharmacology, 2013. 38(10): p. 2027-2034.

23. Titterness, A.K., et al., Voluntary exercise does not enhance long-term potentiation in the adolescent female dentate gyrus. Neuroscience, 2011. 183: p. 25-31.

24. Gomes da Silva, S. and R.M. Arida, Physical activity and brain development. Expert Rev Neurother, 2015. 15(9): p. 1041-51.

25. Gomes da Silva, S., et al., Early exercise promotes positive hippocampal plasticity and improves spatial memory in the adult life of rats. Hippocampus, 2012. 22(2): p. 347-58.

26. Fernandes, J., R.M. Arida, and F. Gomez-Pinilla, Physical exercise as an epigenetic modulator of brain plasticity and cognition. Neurosci Biobehav Rev, 2017. 80: p. 443-456.

27. Butler, C.W., et al., Exercise opens a temporal window for enhanced cognitive improvement from subsequent physical activity. Learn Mem, 2019. 26(12): p. 485-492.

28. Roh, H.C., et al., Simultaneous Transcriptional and Epigenomic Profiling from Specific Cell Types within Heterogeneous Tissues In Vivo. Cell Rep, 2017. 18(4): p. 1048-1061.

29. Deal, R.B. and S. Henikoff, The INTACT method for cell type-specific gene expression and chromatin profiling in Arabidopsis thaliana. Nat Protoc, 2011. 6(1): p. 56-68.

30. Heiman, M., et al., A translational profiling approach for the molecular characterization of CNS cell types. Cell, 2008. 135(4): p. 738-48.

31. Gorski, J.A., et al., Cortical excitatory neurons and glia, but not GABAergic neurons, are produced in the Emx1-expressing lineage. J Neurosci, 2002. 22(15): p. 6309-14.

32. Chan, C.H., et al., Emx1 is a marker for pyramidal neurons of the cerebral cortex. Cereb Cortex, 2001. 11(12): p. 1191-8.

33. Willaime-Morawek, S. and D. van der Kooy, Cortex- and striatum- derived neural stem cells produce distinct progeny in the olfactory bulb and striatum. Eur J Neurosci, 2008. 27(9): p. 2354-62.

34. Luo, L., et al., Optimizing Nervous System-Specific Gene Targeting with Cre Driver Lines: Prevalence of Germline Recombination and Influencing Factors. Neuron, 2020. 106(1): p. 37-65.e5. 
35. Skene, P.J., J.G. Henikoff, and S. Henikoff, Targeted in situ genome-wide profiling with high efficiency for low cell numbers. Nature Protocols, 2018. 13(5): p. 1006-1019.

36. Liu, Y., et al., Glial fibrillary acidic protein-expressing neural progenitors give rise to immature neurons via early intermediate progenitors expressing both glial fibrillary acidic protein and neuronal markers in the adult hippocampus. Neuroscience, 2010. 166(1): p. 241-51.

37. Garcia, A.D.R., et al., GFAP-expressing progenitors are the principal source of constitutive neurogenesis in adult mouse forebrain. Nature Neuroscience, 2004. 7(11): p. 1233-1241.

38. Chucair-Elliott, A.J., et al., Inducible cell-specific mouse models for paired epigenetic and transcriptomic studies of microglia and astroglia. bioRxiv, 2019: p. 862722.

39. Berchtold, N.C., et al., Exercise primes a molecular memory for brain-derived neurotrophic factor protein induction in the rat hippocampus. Neuroscience, 2005. 133(3): p. 853-61.

40. McNulty, S.E., et al., Differential roles for Nr4a1 and Nr4a2 in object location vs. object recognition long-term memory. Learn Mem, 2012. 19(12): p. 588-92.

41. Mi, H. and P. Thomas, PANTHER Pathway: An Ontology-Based Pathway Database Coupled with Data Analysis Tools. 2009, Humana Press. p. 123-140.

42. Krämer, A., et al., Causal analysis approaches in Ingenuity Pathway Analysis. Bioinformatics, 2014. 30(4): p. 523-530.

43. Kandel, E.R., The molecular biology of memory: cAMP, PKA, CRE, CREB-1, CREB-2, and CPEB. Molecular Brain, 2012. 5(1): p. 14.

44. Kerimoglu, C., et al., KMT2A and KMT2B Mediate Memory Function by Affecting Distinct Genomic Regions. Cell Reports, 2017. 20(3): p. 538-548.

45. Ashbrook, D.G., et al., Transcript co-variance with Nestin in two mouse genetic reference populations identifies Lef1 as a novel candidate regulator of neural precursor cell proliferation in the adult hippocampus. Front Neurosci, 2014. 8: p. 418.

46. Tapia-González, S., et al., Smad3 is required for the survival of proliferative intermediate progenitor cells in the dentate gyrus of adult mice. Cell Communication and Signaling, 2013. 11(1): p. 93.

47. Tiano, J.P., D.A. Springer, and S.G. Rane, SMAD3 Negatively Regulates Serum Irisin and Skeletal Muscle FNDC5 and Peroxisome Proliferator-activated Receptor $\gamma$ Coactivator 1- $\alpha$ (PGC-1 $\alpha)$ during Exercise. Journal of Biological Chemistry, 2015. 290(12): p. 7671-7684.

48. Sun, Z., et al., EGR1 recruits TET1 to shape the brain methylome during development and upon neuronal activity. Nature Communications, 2019. $\mathbf{1 0}(1)$.

49. Bouché, V., et al., Drosophila Mitf regulates the V-ATPase and the lysosomalautophagic pathway. Autophagy, 2016. 12(3): p. 484-98.

50. Guan, Z., et al., Integration of long-term-memory-related synaptic plasticity involves bidirectional regulation of gene expression and chromatin structure. Cell, 2002. 111(4): p. 483-93. 
51. Korzus, E., M.G. Rosenfeld, and M. Mayford, CBP Histone Acetyltransferase Activity Is a Critical Component of Memory Consolidation. Neuron, 2004. 42(6): p. 961-972.

52. Subramanian, A., et al., Gene set enrichment analysis: A knowledge-based approach for interpreting genome-wide expression profiles. Proceedings of the National Academy of Sciences, 2005. 102(43): p. 15545-15550.

53. Nofrini, V., D. Di Giacomo, and C. Mecucci, Nucleoporin genes in human diseases. European Journal of Human Genetics, 2016. 24(10): p. 1388-1395.

54. Gomes-Duarte, A., et al., eIF3: a factor for human health and disease. RNA Biol, 2018. 15(1): p. 26-34.

55. Alvarez-López, M.J., et al., Long-term exercise modulates hippocampal gene expression in senescent female mice. J Alzheimers Dis, 2013. 33(4): p. 117790.

56. Butler, A.A., et al., O-GlcNAc and EZH2-mediated epigenetic regulation of gene expression during consolidation of fear memories. Learning \& memory (Cold Spring Harbor, N.Y.), 2019. 26(9): p. 373-379.

57. Butler, A.A., et al., O-GlcNAc and EZH2-mediated epigenetic regulation of gene expression during consolidation of fear memories. Learn Mem, 2019. 26(9): p. 373-379.

58. Kaya-Okur, H.S., et al., CUT\&Tag for efficient epigenomic profiling of small samples and single cells. Nature Communications, 2019. 10(1).

59. Stergiopoulos, A., M. Elkouris, and P.K. Politis, Prospero-related homeobox 1 (Prox1) at the crossroads of diverse pathways during adult neural fate specification. Front Cell Neurosci, 2014. 8: p. 454.

60. Camp, N.D., et al., Individual protomers of a $G$ protein-coupled receptor dimer integrate distinct functional modules. Cell Discov, 2015. 1: p. 15011-.

61. Baple, E.L., et al., Mutations in KPTN cause macrocephaly, neurodevelopmental delay, and seizures. Am J Hum Genet, 2014. 94(1): p. 87-94.

62. Peterson, M.D. and M.A. Titus, F-actin distribution of Dictyostelium myosin I double mutants. J Eukaryot Microbiol, 1994. 41(6): p. 652-7.

63. Johnson Chacko, L., et al., Transcriptome-Wide Analysis Reveals a Role for Extracellular Matrix and Integrin Receptor Genes in Otic Neurosensory Differentiation from Human iPSCs. Int J Mol Sci, 2021. 22(19).

64. Truter, S., et al., Pro-alpha 2(V) collagen gene; pairwise analysis of the aminopropeptide coding domain, and cross-species comparison of the promoter sequence. Connect Tissue Res, 1993. 29(1): p. 51-9.

65. Lévy, J., et al., EFNB2 haploinsufficiency causes a syndromic neurodevelopmental disorder. Clin Genet, 2018. 93(6): p. 1141-1147.

66. Xiong, C., et al., Targeting Forward and Reverse EphB4/EFNB2 Signaling by a Peptide with Dual Functions. Sci Rep, 2020.10(1): p. 520.

67. Zhu, F., et al., EFNB2 facilitates cell proliferation, migration, and invasion in pancreatic ductal adenocarcinoma via the p53/p21 pathway and EMT. Biomed Pharmacother, 2020. 125: p. 109972.

68. Ferrari, K.J., E. Lavarone, and D. Pasini, The Dual Role of EPOP and Elongin BC in Controlling Transcriptional Activity. Mol Cell, 2016. 64(4): p. 637-638. 
69. Liefke, R., V. Karwacki-Neisius, and Y. Shi, EPOP Interacts with Elongin BC and USP7 to Modulate the Chromatin Landscape. Mol Cell, 2016. 64(4): p. 659-

672.

70. Jordan, J.T., The rodent hippocampus as a bilateral structure: A review of hemispheric lateralization. Hippocampus, 2020. 30(3): p. 278-292.

71. Iwano, T., et al., Prox1 postmitotically defines dentate gyrus cells by specifying granule cell identity over CA3 pyramidal cell fate in the hippocampus. Development, 2012. 139(16): p. 3051-62.

72. Luo, C., et al., Single-cell methylomes identify neuronal subtypes and regulatory elements in mammalian cortex. Science, 2017. 357(6351): p. 600-604.

73. Skene, P.J., J.G. Henikoff, and S. Henikoff, Targeted in situ genome-wide profiling with high efficiency for low cell numbers. Nat Protoc, 2018. 13(5): p. 1006-1019.

74. Helbling, J.-C., et al., Combined Gene Expression and Chromatin Immunoprecipitation From a Single Mouse Hippocampus. Current Protocols, 2021. 1(2): p. e33.

75. Chucair-Elliott, A.J., et al., Inducible cell-specific mouse models for paired epigenetic and transcriptomic studies of microglia and astroglia. Commun Biol, 2020. 3(1): p. 693.

76. Doty, R.W., et al., Long-term reversal of hemispheric specialization for visual memory in a split-brain macaque. Behav Brain Res, 1999. 102(1-2): p. 99113.

77. Duboc, V., et al., Asymmetry of the Brain: Development and Implications. Annu Rev Genet, 2015. 49: p. 647-72.

78. Güntürkün, O., F. Ströckens, and S. Ocklenburg, Brain Lateralization: A Comparative Perspective. Physiol Rev, 2020. 100(3): p. 1019-1063.

79. Hubel, D.H. and T.N. Wiesel, Receptive fields, binocular interaction and functional architecture in the cat's visual cortex. The Journal of Physiology, 1962. 160(1): p. 106-154.

80. Spiers, H.J., et al., Unilateral temporal lobectomy patients show lateralized topographical and episodic memory deficits in a virtual town. Brain, 2001. 124(Pt 12): p. 2476-89.

81. Long, J.Z., et al., A smooth muscle-like origin for beige adipocytes. Cell Metab, 2014. 19(5): p. 810-20.

82. Deal, R.B. and S. Henikoff, A simple method for gene expression and chromatin profiling of individual cell types within a tissue. Dev Cell, 2010. 18(6): p. 103040.

83. Pfaffl, M.W., A new mathematical model for relative quantification in real-time RT-PCR. Nucleic Acids Res, 2001. 29(9): p. e45.

84. Schwarz, J.M., Using fluorescence activated cell sorting to examine cell-typespecific gene expression in rat brain tissue. J Vis Exp, 2015(99): p. e52537.

85. Andrews, S., FastQC, S. Andrews, Editor. 2005, Babraham Bioinformatics.

86. Dobin, A., et al., STAR: ultrafast universal RNA-seq aligner. Bioinformatics, 2013. 29(1): p. 15-21.

87. Broad Institute, Picard. 2013, Broad Institute. 
88. R Development Core Team, R. 2010, R Foundation for statistical Computing: Vienna, Austria. p. A language and environment for statistical computing.

89. Lawrence, M., et al., Software for Computing and Annotating Genomic Ranges. PLoS Computational Biology, 2013. 9(8): p. e1003118.

90. Martin Morgan, H.P., Valerie Obenchain, Nathaniel Hayden, Rsamtools: Binary alignment (BAM), FASTA, variant call (BCF), and tabix file import. 2021, Bioconductor Bioconductor

91. Love, M.I., W. Huber, and S. Anders, Moderated estimation of fold change and dispersion for RNA-seq data with DESeq2. Genome Biology, 2014. 15(12).

92. Durinck, S., et al., BioMart and Bioconductor: a powerful link between biological databases and microarray data analysis. Bioinformatics, 2005. 21(16): p. 3439-3440.

93. Durinck, S., et al., Mapping identifiers for the integration of genomic datasets with the R/Bioconductor package biomaRt. Nature Protocols, 2009. 4(8): p. 1184-1191.

94. Wickham, H., Ggplot2: Elegant graphics for data analysis, in Use R! 2016, Springer International Publishing: Cham, Switzerland.

95. Gregory R. Warnes, B.B., Lodewijk Bonebakker, Robert Gentleman, Wolfgang Huber, Andy Liaw, Thomas Lumley, Martin Maechler, Arni Magnusson, Steffen Moeller, Marc Schwartz, Bill Venables, Tal Galili, gplots: Various $R$ Programming Tools for Plotting Data. 2020, Tal Galili

96. Neuwirth, E., RColorBrewer: ColorBrewer Palettes. 2014, Erich Neuwirth: CRAN.

97. Bioinformatics \& Evolutionary Genomics, Venn Diagram: Calculate and draw custom Venn diagrams. 2021: BELGIUM.

98. Langmead, B. and S.L. Salzberg, Fast gapped-read alignment with Bowtie 2. Nature Methods, 2012. 9(4): p. 357-359.

99. Humburg, P., et al., ChIPseqR: analysis of ChIP-seq experiments. BMC Bioinformatics, 2011. 12(1): p. 39.

100. Bioconductor Core Team, B.P.M., TxDb.Mmusculus.UCSC.mm10.knownGene: Annotation package for TxDb object(s). 2019, Bioconductor

101. Kent, W.J., et al., The Human Genome Browser at UCSC. Genome Research, 2002. 12(6): p. 996-1006.

102. Rosenbloom, K.R., et al., ENCODE whole-genome data in the UCSC Genome Browser. Nucleic Acids Research, 2010. 38(Database): p. D620-D625.

103. Haeussler, M., et al., Navigating protected genomics data with UCSC Genome Browser in a Box. Bioinformatics, 2014. 31(5): p. 764-766.

104. Kassambara, A., ggpubr: 'ggplot2' Based Publication Ready Plots, A. Kassambara, Editor. 2020, CRAN. 
bioRxiv preprint doj: https://doi.grg/10.1101/2021.12.23.473936; this version posted December 23, 2021. The copyright holder for this preprint

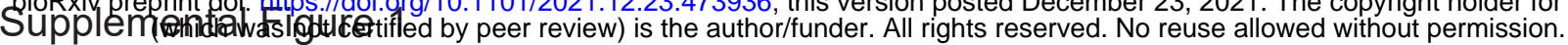
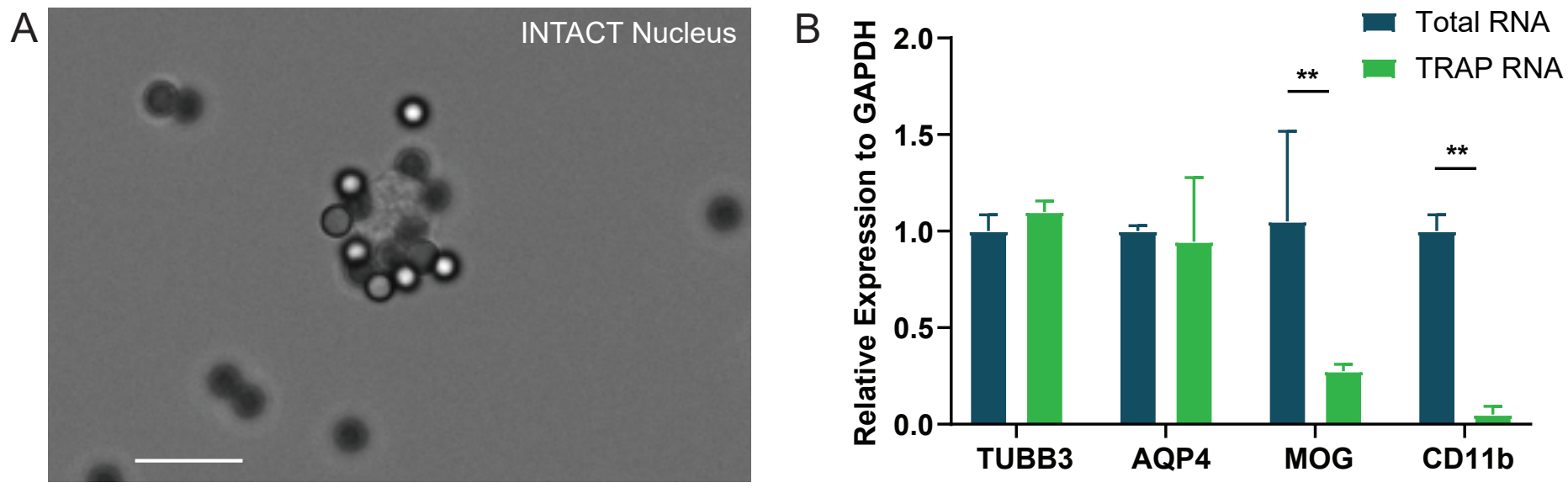

C

Daily Running Distances by Cage During ELE

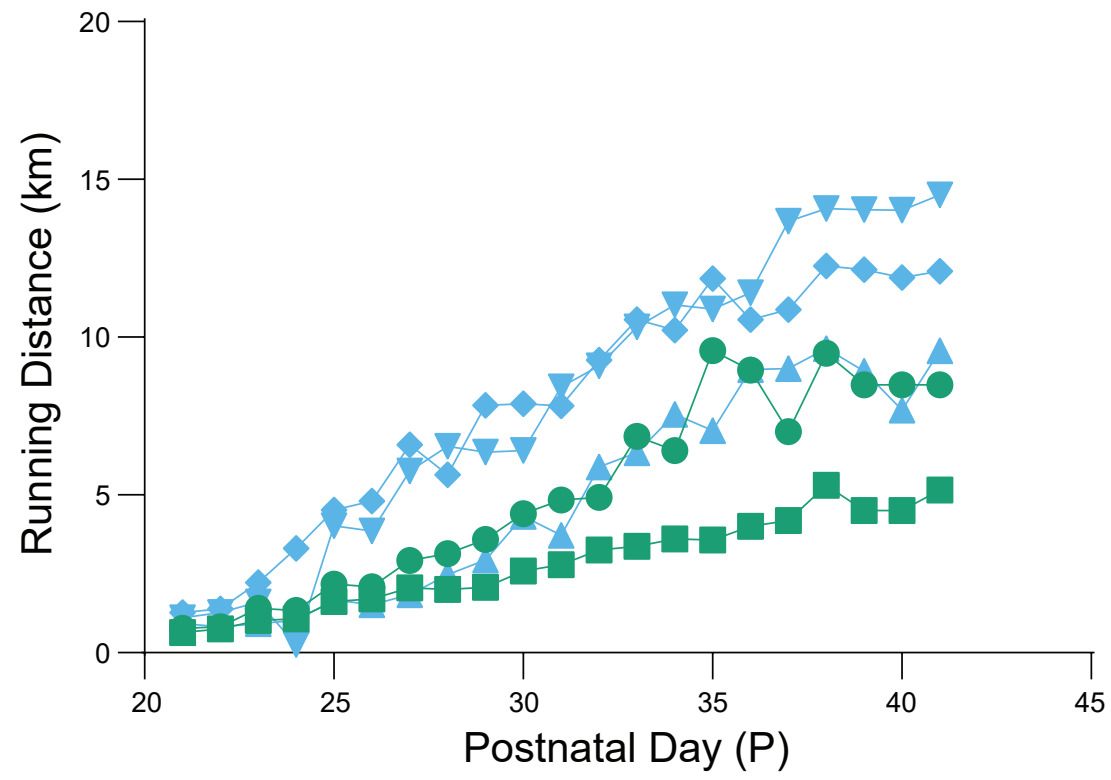

Separate Isolation Group

$\rightarrow \quad$ TG9.1.3-TG9.1.4

$-\quad$ TG9.2.1-TG9.2.2

$\triangle \quad$ TG7.3.1-TG7.3.2

Simultaneous Isolation Group

-- NT6.303-NT6.304

TG7.4.1-TG7.4.2 
Supplemental Figure 1: (A) Brightfield microscopy of a neural nucleus bound to streptavidin coated Dynabeads ${ }^{\mathrm{TM}}$. The scale bar represents $10 \mu \mathrm{m}$. (B) qPCR of TRAP isolated RNA compared to total RNA from the simultaneous isolation protocol $* * \mathrm{p}<0.01$. (C) Daily running distances of each cage during the ELE paradigm. Values of days missing or low due to software error were imputed by averaging the previous 3 days available. 2 Way Analysis of Variance (ANOVA) reveals that there is significant difference between postnatal days $(\mathrm{p}<0.0001)$ and cages $(\mathrm{p}<0.0001)$ but no significant effect of isolation $(\mathrm{p}=0.1508)$. 
bioRxiv preprint doj: https://doi.grg/10.1101/2021.12.23.473936; this version posted December 23, 2021. The copyright holder for this preprint

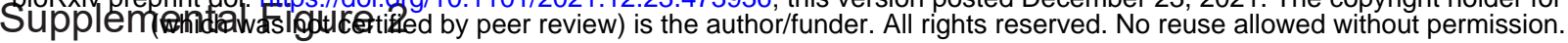
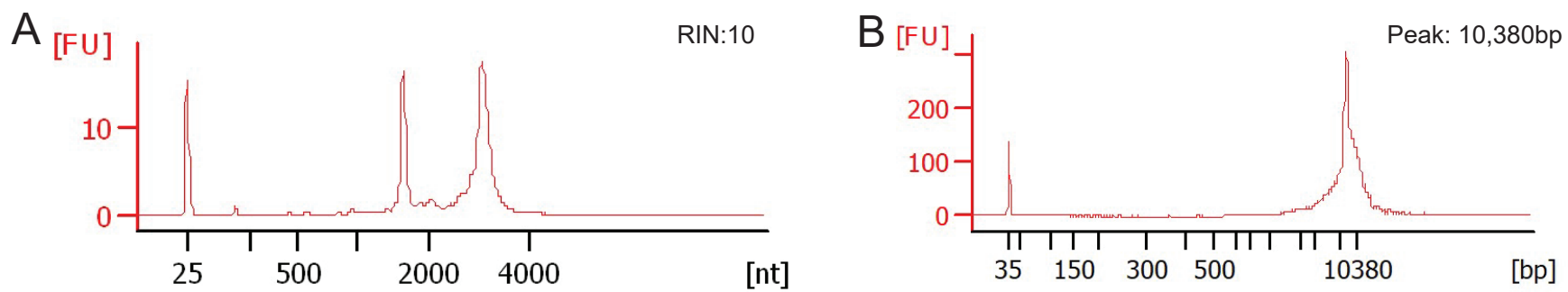

C

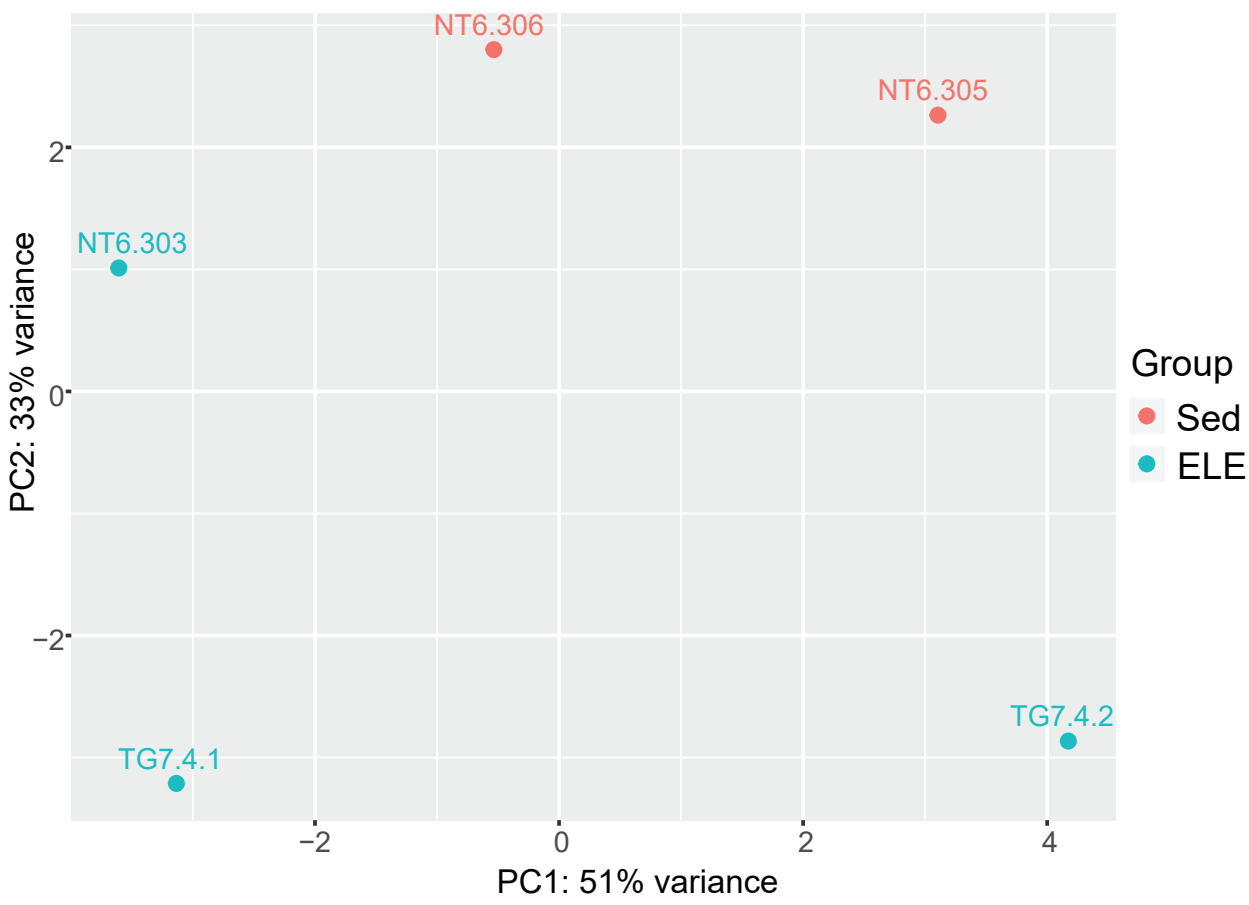

D

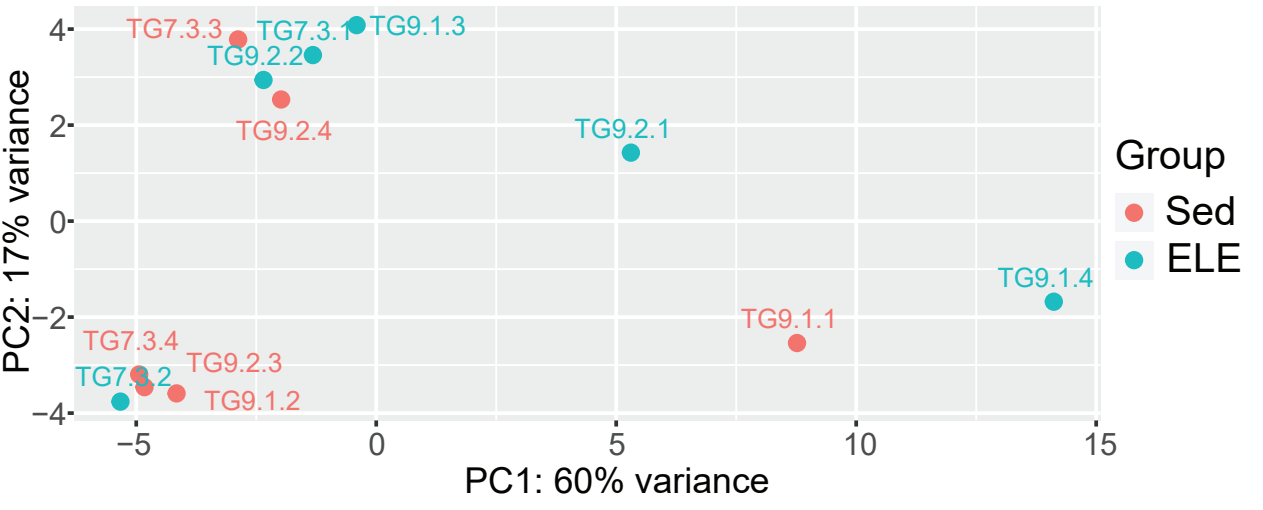


Supplemental Figure 2: (A) Representative electropherogram of RNA extracted using the simultaneous isolation protocol. (B) Representative electropherogram of DNA extracted using the simultaneous isolation protocol. (C) Principle component analysis plot (PCA) of RNA-seq data for the simultaneous isolations. (D) PCA of RNA-seq data for the separate isolations. 
bioRxiv preprint doi: https://doi.grg/10.1101/2021.12.23.473936; this version posted December 23, 2021. The copyright holder for this preprint

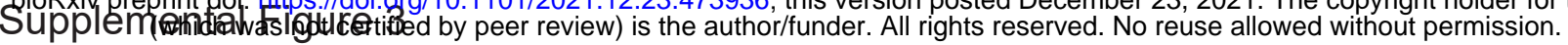

A

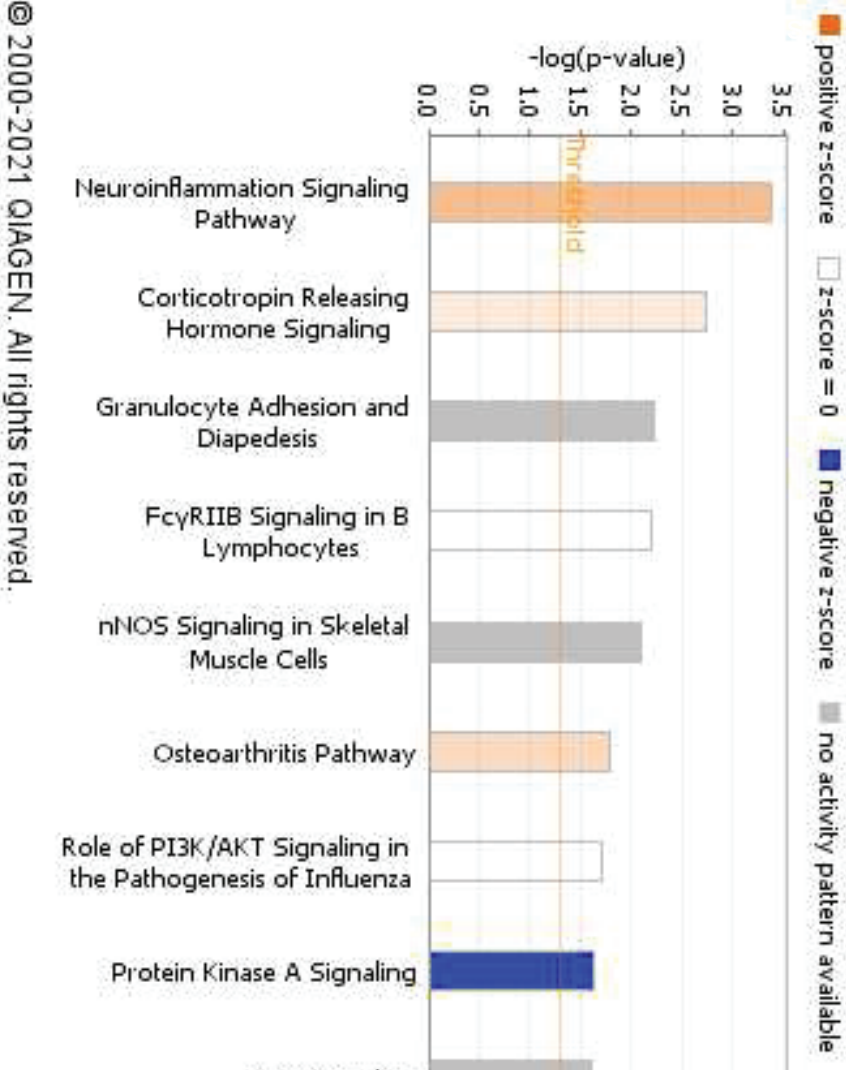


bioRxiv preprint doi: https://doi.org/10.1101/2021.12.23.473936; this version posted December 23, 2021. The copyright holder for this preprint (which was not certified by peer review) is the author/funder. All rights reserved. No reuse allowed without permission.

Supplemental Figure 3: (A) Canonical pathways identified by Qiagen's IPA of genes upregulated by ELE. Supplemental table 3C and 3D have the genes identified by the top two pathways listed in this bar chart. 